\title{
Low-rank filter and detector for multidimensional data based on an alternative unfolding HOSVD: application to polarimetric STAP
}

\author{
Maxime Boizard ${ }^{1}{ }^{2 *}$, Guillaume Ginolhac ${ }^{3}$, Fréderic Pascal ${ }^{2}$ and Philippe Forster ${ }^{1}$
}

\begin{abstract}
This paper proposes an extension of the higher order singular value decomposition (HOSVD), namely the alternative unfolding HOSVD (AU-HOSVD), in order to exploit the correlated information in multidimensional data. We show that the properties of the AU-HOSVD are proven to be the same as those for HOSVD: the orthogonality and the low-rank (LR) decomposition. We next derive LR filters and LR detectors based on AU-HOSVD for multidimensional data composed of one LR structure contribution. Finally, we apply our new LR filters and LR detectors in polarimetric space-time adaptive processing (STAP). In STAP, it is well known that the response of the background is correlated in time and space and has a LR structure in space-time. Therefore, our approach based on AU-HOSVD seems to be appropriate when a dimension (like polarimetry in this paper) is added. Simulations based on signal-to-interferenceplus-noise ratio (SINR) losses, probability of detection (Pd), and probability of false alarm (Pfa) show the interest of our approach: LR filters and LR detectors which can be obtained only from AU-HOSVD outperform the vectorial approach and those obtained from a single HOSVD.
\end{abstract}

Keywords: Multilinear algebra; HOSVD; Low-rank approximation; STAP; Low-rank filter; Low-rank detector

\section{Introduction}

In signal processing, more and more applications deal with multidimensional data, whereas most of the signal processing algorithms are derived based on oneor two-dimensional models. Consequently, multidimensional data have to be folded as vector or matrix to be processed. These operations are not lossless since they involve a loss of structure. Several issues may arise from this loss: decrease of performances and lack of robustness (see for instance [1]). The multilinear algebra [2,3] provides a good framework to exploit these data by preserving the structure information. In this context, data are represented as multidimensional arrays called tensor. However, generalizing matrix-based algorithms to the multilinear algebra framework is not a trivial task. In particular, some multilinear tools do not retain all the properties of the vectorial and matrix tools. Let us consider the case of the

\footnotetext{
${ }^{*}$ Correspondence: maxime.boizard@lss.supelec.fr

1 SATIE, ENS Cachan, 61, av President Wilson, Cachan 94230, France

2 SONDRA, Supelec, 3 rue Joliot-Curie, Gif-sur-Yvette 91192, France

Full list of author information is available at the end of the article
}

singular value decomposition (SVD). The SVD decomposes a matrix into a sum of rank-1 matrices and has uniqueness and orthonormality properties. There is no single multilinear extension of the SVD, with exactly the same properties as the SVD. Depending on which properties are preserved, several extensions of the SVD have been introduced.

On one hand, CANDECOMP/PARAFAC (CP) [4] decomposes a tensor as a sum of rank- 1 tensors, preserving the definition of rank. Due to its properties of identifiability and uniqueness, this decomposition is relevant for multiple parameter estimation. CP was first introduced in the signal processing community for direction of arrival (DOA) estimation $[5,6]$. New decompositions were then derived from CP. For example, in [7,8], a decomposition based on a constrained CP model is applied to multipleinput multiple-output (MIMO) wireless communication system. These decompositions share a common issue for some applications: they are not orthogonal.

On the other hand, the higher order singular value decomposition (HOSVD) [3,9] decomposes a tensor as a product of a core tensor and a unitary matrix for each 
dimension of the tensor. This decomposition relies on matrix unfoldings. In general, HOSVD does not have the properties of identifiability and uniqueness. The model provided by HOSVD is defined up to a rotation. It implies that HOSVD cannot be used for multiple parameters estimation unlike CP. Orthogonality properties of HOSVD allow to extend the low-rank methods such as $[10,11]$. These methods are based on the ranks of the different matrix unfoldings, called $p$-ranks $[2,12,13]$. HOSVD has been successfully applied in many fields such as image processing [14], sonar and seismo-acoustic [15], ESPRIT [16], ICA [17], and video compression [18].

HOSVD is based on the classic tensor unfolding and in particular on the matrix of left singular vectors. This unfolding transforms a tensor into a matrix in order to highlight one dimension. In other words, HOSVD only considers simple information, which is the information contained in each dimension taken separately. The correlated information, which is the information contained in a combination of dimensions, is neglected. In [19], a new decomposition, PARATREE ${ }^{\text {a }}$, based on the sequential unfolding SVD (SUSVD) was proposed. This decomposition considers some correlated information, using the right matrix of the singular vectors. This approach can be improved, to consider any type of correlated information. Consequently, we propose to develop a new set of orthogonal decompositions which will be called alternative unfolding HOSVD (AU-HOSVD). In this paper, we will define this new operator and study its main properties, especially the extension of the low-rank approximation. We will show the link between AU-HOSVD and HOSVD.

Based on this new decomposition, we derive new lowrank (LR) filters and LR detectors for multidimensional data containing a target embedded in an interference. We assume that the interference is the sum of two noises: a white Gaussian noise and a low-rank structured one. In order to illustrate the interest of these new LR filters and LR detectors, we will consider the multidimensional space-time adaptive processing (STAP). STAP is a technique used in airborne phased array radar to detect moving target embedded in an interference background such as jamming (jammers are not considered in this paper) or strong ground clutter [20] plus a white Gaussian noise (resulting from the sensor noise). While conventional radars are capable of detecting targets both in the time domain related to target range and in the frequency domain related to target velocity, STAP uses an additional domain (space) related to the target angular localization. From the Brennan rule [21], STAP clutter is shown to have a low-rank structure ${ }^{\mathrm{b}}$. That means that the clutter response in STAP is correlated in time and space. Therefore, if we add a dimension, the LR filter and LR detector based on HOSVD will not be interesting. In this paper, we show the interest of our new LR filters and LR detectors based on AU-HOSVD in a particular case of multidimensional STAP: polarimetric STAP [22]. In this polarimetric configuration, each element transmits and receives in both $\mathrm{H}$ and $\mathrm{V}$ polarizations, resulting in three polarimetric channels $(\mathrm{HH}, \mathrm{VV}, \mathrm{HV} / \mathrm{VH})$. The dimension of the data model is then three. Simulations based on signal-to-interference-plus-noise ratio (SINR) losses [20], probability of detection (Pd), and probability of false alarm (Pfa) show the interest of our approach: LR filters and LR detectors which are obtained using AU-HOSVD outperform the vectorial approach and those obtained from HOSVD in the general polarimetry model (the channels $\mathrm{HH}$ and $\mathrm{VV}$ are not completely correlated). We believe that these results could be extended to more generalized multidimensional STAP systems like MIMO-STAP [23-26].

The paper is organized as follows. Section 2 gives a brief overview of the basic multilinear algebra tools. In particular, the HOSVD and its main properties are presented. In Section 3, the AU-HOSVD and its properties are derived. Section 4 is devoted to the derivation of the LR filters and LR detectors based on AU-HOSVD. Finally, in Section 5, these new tools are applied to the case of polarimetric STAP.

The following convention is adopted: scalars are denoted as italic letters, vectors as lowercase boldface letters, matrices as boldface capitals, and tensors as boldface calligraphic letters. We use the superscripts ${ }^{H}$ for Hermitian transposition and ${ }^{*}$ for complex conjugation. The expectation is denoted by $E[$.$] , and the Frobenius norm is$ denoted $\|$.$\| .$

\section{Some basic multilinear algebra tools}

This section contains the main multilinear algebra tools used in this paper. Let $\mathcal{H}, \mathcal{B} \in \mathbb{C}^{I_{1} \times \ldots \times I_{P}}$, be two Pth-order tensors and $h_{i_{1} \ldots i_{P}}, b_{i_{1} \ldots i_{P}}$ their elements.

\subsection{Basic operators of multilinear algebra}

Unfoldings In this paper, three existing unfoldings are used; for a general definition of tensor unfolding, we refer the reader to [2].

- Vector: vec transforms a tensor $\mathcal{H}$ into a vector, $\operatorname{vec}(\mathcal{H}) \in \mathbb{C}^{I_{1} I_{2} \ldots I_{P}}$. We denote $\operatorname{vec}^{-1}$, the inverse operator.

- Matrix: this operator transforms the tensor $\mathcal{H}$ into a matrix $[\mathcal{H}]_{p} \in \mathbb{C}^{I_{p} \times I_{1} \ldots I_{p-1} I_{p+1} \ldots I_{P}}, p=1 \ldots P$. For example, $[\mathcal{H}]_{1} \in \mathbb{C}^{I_{1} \times I_{2} \ldots I_{P}}$. This transformation allows to enhance simple information (i.e., information contained in one dimension of the tensor).

- Square matrix: this operator transforms a $2 P$ th-order tensor $\mathcal{R} \in \mathbb{C}^{I_{1} \times I_{2} \ldots \times I_{P} \times I_{1} \times I_{2} \ldots \times I_{P}}$ into a square 
matrix, $\operatorname{SqMat}(\mathcal{R}) \in \mathbb{C}^{I_{1} \ldots I_{P} \times I_{1} \ldots I_{P}} . S q M a t^{-1}$ is the inverse operator.

The inverse operators always exist. However, the way the tensor was unfolded must be known.

\section{Products}

- The scalar product $\langle\mathcal{H}, \mathcal{B}>$ of two tensors is defined as

$$
\begin{aligned}
<\mathcal{H}, \mathcal{B}> & =\sum_{i_{1}} \sum_{i_{2}} \ldots \sum_{i_{P}} b_{i_{1} i_{2} \ldots i_{P}}^{*} h_{i_{1} i_{2} \ldots i_{P}} \\
& =\operatorname{vec}(\mathcal{B})^{H} \operatorname{vec}(\mathcal{H}) .
\end{aligned}
$$

It is the usual Hermitian scalar product in linear spaces [12,27].

- Let $\mathbf{E} \in \mathbb{C}^{J_{n} \times I_{n}}$ be a matrix, the $n$-mode product between $\mathbf{E}$ and a tensor $\mathcal{H}$ is defined as

$$
\begin{array}{rc} 
& \mathcal{G}=\mathcal{H} \times{ }_{n} \mathbf{E} \in \mathbb{C}^{I_{1} \times \ldots \times J_{n} \times \ldots \times I_{P}} \\
\Longleftrightarrow & (\mathcal{G})_{i_{1} \ldots j_{n} \ldots i_{P}}=\sum_{i_{n}} h_{i_{1} \ldots i_{n} \ldots i_{P}} e_{j_{n} i_{n}} \\
\Longleftrightarrow & {[\mathcal{G}]_{n}=\mathbf{E}[\mathcal{H}]_{n} .}
\end{array}
$$

- The outer product between $\mathcal{H}$ and $\mathcal{B}$, $\mathcal{E}=\mathcal{H} \circ \mathcal{B} \in \mathbb{C}^{I_{1} \times \ldots \times I_{P} \times I_{1} \times \ldots \times I_{P}}$ is defined as

$$
e_{i_{1} \ldots i_{P} i_{1} \ldots i_{P}}=h_{i_{1} \ldots i_{P}} . b_{i_{1} \ldots i_{P}} .
$$

Tensor ranks There are two concepts of rank for tensors:

- The tensor rank: it is defined as the minimum number of rank-1 tensors (for example a Pth-order rank-1 tensor can be written as the outer product of $P$ vectors) necessary to obtain the considered tensor.

- The $p$-ranks: they are defined as the ranks of the unfoldings, $r_{p}=\operatorname{rank}\left([\mathcal{H}]_{p}\right)$.

\subsection{Higher order singular value decomposition}

This subsection recalls the main results on the HOSVD used in this paper.

Theorem 2.1. The higher order singular value decomposition (HOSVD) is a particular case of Tucker decomposition [9] with orthogonality properties. HOSVD decomposes a tensor $\mathcal{H}$ as follows [3]:

$$
\mathcal{H}=\mathcal{K} \times{ }_{1} \mathbf{U}^{(1)} \ldots \times_{P} \mathbf{U}^{(P)}
$$

where $\forall n, \mathbf{U}^{(n)} \in \mathbb{C}^{I_{n} \times I_{n}}$ is an orthonormal matrix and $\mathcal{K} \in \mathbb{C}^{I_{1} \times \ldots \times I_{P}}$ is the core tensor, which satisfies the all-orthogonality conditions [3]. The matrix $\mathbf{U}^{(n)}$ is given by the singular value decomposition of the $n$-dimension unfolding, $[\mathcal{H}]_{n}=\mathbf{U}^{(n)} \Sigma^{(n)} \mathbf{V}^{(n) H}$. Using classical unfolding, the HOSVD only considers the simple information.

Remark Let $\mathcal{H} \in \mathbb{C}^{I_{1} \times I_{2} \ldots \times I_{P} \times I_{1} \times I_{2} \ldots \times I_{P}}$ be a $2 P$ th-order Hermitian tensor, i.e., $h_{i_{1}, \ldots, i_{p}, j_{1}, \ldots, j_{p}}=h_{j_{1}, \ldots, j_{p}, i_{1}, \ldots, i_{p}}^{*}$. The HOSVD of $\mathcal{H}$ is written as [16]

$$
\mathcal{H}=\mathcal{K} \times{ }_{1} \mathbf{U}^{(1)} \ldots \times{ }_{P} \mathbf{U}^{(P)} \times{ }_{P+1} \mathbf{U}^{(1) *} \ldots \times{ }_{2 P} \mathbf{U}^{(P) *} .
$$

The following result permits to compute a tensor approximation of lower $p$-ranks.

Proposition 2.1 (Low-rank approximation). Let us introduce $\mathcal{H}=\mathcal{H}_{c}+\mathcal{H}_{0} . \mathcal{H}_{c}$ is a $\left(r_{1}, \ldots, r_{P}\right)$ low-rank tensor where $r_{k}<I_{k}$, for $k=1, \ldots, P$. An approximation of $\mathcal{H}_{0}$ is given by $[15,27]$ :

$$
\begin{aligned}
& \qquad \mathcal{H}_{0} \approx \mathcal{H} \times{ }_{1} \mathbf{U}_{0}^{(1)} \mathbf{U}_{0}^{(1) H} \ldots \times_{P} \mathbf{U}_{0}^{(P)} \mathbf{U}_{0}^{(P) H}, \\
& \text { with } \mathbf{U}_{0}^{(n)}=\left[\mathbf{u}_{r_{n}+1}^{(n)} \ldots \mathbf{u}_{I_{n}}^{(n)}\right] .
\end{aligned}
$$

It is well known that this solution is not optimal in the sense of least squares. However, it is a correct approximation in most cases $[16,27]$ and it is easy to implement. That is why iterative algorithms will not be used in this paper.

\subsection{Covariance tensor and estimation}

Definition Let $\mathcal{Z} \in \mathbb{C}^{I_{1} \times \ldots \times I_{P}}$ be a random $P$ th-order tensor, and the covariance tensor $\mathcal{R} \in \mathbb{C}^{I_{1} \times \ldots \times I_{P} \times I_{1} \ldots \times I_{P}}$ is defined as [28]

$$
\mathcal{R}=E\left[z \circ z^{*}\right] \text {. }
$$

Sample covariance matrix Let $\mathbf{z} \in \mathbb{C}^{I_{1} \ldots I_{P}}$ be a zeromean Gaussian random vector and $\mathbf{R} \in \mathbb{C}^{I_{1} \ldots I_{P} \times I_{1} \ldots I_{P}}$ its covariance matrix. Let $\mathbf{z}_{k}$ be $K$ observations of $\mathbf{z}$. The sample covariance matrix (SCM), $\hat{\mathbf{R}}$, is written as follows:

$$
\hat{\mathbf{R}}=\frac{1}{K} \sum_{k=1}^{K} \mathbf{z}_{k} \mathbf{z}_{k}^{H} .
$$

Sample covariance tensor Let $\boldsymbol{Z}_{k} \in \mathbb{C}^{I_{1} \times \ldots \times I_{P}}$ be $K$ observations of $\mathcal{Z}$. By analogy with the SCM, $\hat{\mathcal{R}} \in$ $\mathbb{C}^{I_{1} \ldots \times I_{P} \times I_{1} \ldots \times I_{P}}$, the sample covariance tensor $(\mathrm{SCT})$ is defined as [16]

$$
\hat{\mathcal{R}}=\frac{1}{K} \sum_{k=1}^{K} z_{k} \circ z_{k}^{*} .
$$

Remark If we denote $\mathbf{z}=\operatorname{vec}(\boldsymbol{Z})$, then

$$
\mathbf{R}=\operatorname{SqMat}(\mathcal{R}) .
$$

\section{Alternative unfolding HOSVD}

Due to proposition 2.1, it is possible to design LR filters based on HOSVD. This approach does not work when all $p$-ranks are full (i.e., $r_{p}=I_{p}, p=1 \ldots P$ ), since no projection could be done. However, the data may still have 
a LR structure. This is the case of correlated data where one or more ranks relative to a group of dimensions are deficient. Tensor decompositions allowing to exploit this kind of structure have not been promoted. To fill this gap, we propose to introduce a new tool which will be able to extract this kind of information. This section contains the main contribution of this paper: the derivation of the AU-HOSVD and its principal properties.

\subsection{Generalization of standard operators}

Notation of indices In order to consider correlated information, we introduce a new notation for the indices of a tensor. We consider $\mathcal{H} \in \mathbb{C}^{I_{1} \times \ldots \times I_{P}}$, a $P$ th-order tensor. We denote $\mathbb{A}=\{1, \ldots, P\}$ the set of the dimensions and $\mathbb{A}_{1}, \ldots, \mathbb{A}_{L}, \mathrm{~L}$ subsets of $\mathbb{A}$ which define a partition of $\mathbb{A}$. In other words, $\mathbb{A}_{1}, \ldots, \mathbb{A}_{L}$ satisfy the following conditions:

- $\mathbb{A}_{1} \cup \ldots \cup \mathbb{A}_{L}=\mathbb{A}$

- They are pairwise disjoint, i.e., $\forall i \neq j, \mathbb{A}_{i} \cap \mathbb{A}_{j}=\emptyset$.

Moreover $\mathbb{C}^{I_{1} \ldots I_{P}}$ is denoted $\mathbb{C}^{I_{\mathbb{A}}}$. For example, when $\mathbb{A}_{1}=$ $\{1,2\}$ and $\mathbb{A}_{2}=\{3,4\}, \mathbb{C}^{I_{\mathbb{A}_{1}} \times I_{\mathbb{A}_{2}}}$ means $\mathbb{C}^{I_{1} I_{2} \times I_{3} I_{4}}$.

A generalization of unfolding in matrices In order to build our new decomposition, we need a generalized unfolding, adapted from [2]. This operator allows to unfold a tensor into a matrix whose dimensions could be any combination $\mathbb{A}_{l}$ of the tensor dimensions. It is denoted as $[.]_{\mathbb{A}_{l}}$, and it transforms $\mathcal{H}$ into a matrix $[\mathcal{H}]_{\mathbb{A}_{l}} \in$

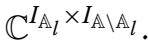

A new unfolding in tensors We denote as Reshape the operator which transforms a tensor $\mathcal{H}$ into a tensor $\operatorname{Reshape}\left(\mathcal{H}, \mathbb{A}_{1}, \ldots, \mathbb{A}_{L}\right) \in \mathbb{C}^{I_{\mathbb{A}_{1}} \times \ldots \times I_{\mathbb{A}_{L}}}$ and Reshape ${ }^{-1}$ the inverse operator.

A new tensor product The $n$-mode product allows to multiply a tensor with a matrix along one dimension. We propose to extend the $n$-mode product to multiply a tensor with a matrix along several dimensions, combined in $\mathbb{A}_{l}$. Let $\mathbf{D} \in \mathbb{C}^{I_{\mathbb{A}_{l}} \times I_{\mathbb{A}_{l}}}$ be a square matrix. This new product, called multimode product, is defined as

$$
\mathcal{B}=\mathcal{H} \times_{\mathbb{A}_{l}} \mathbf{D} \Longleftrightarrow[\mathcal{B}]_{\mathbb{A}_{l}}=\mathbf{D}[\mathcal{H}]_{\mathbb{A}_{l}}
$$

The following proposition shows the link between multimode product and $n$-mode product.

Proposition 3.1 (Link between $n$-mode product and multimode product). Let $\mathcal{H} \in \mathbb{C}^{I_{1} \times \ldots \times I_{P}}$ be a Pth-order tensor, $\mathbb{A}_{1}, \ldots, \mathbb{A}_{L}$ be a partition of $\mathbb{A}$, and $\mathbf{D} \in \mathbb{C}^{I_{\mathbb{A}_{l}} \times I_{\mathbb{A}_{l}} \text { be }}$ a square matrix. Then, the following equality is verified:

$$
\begin{aligned}
& \operatorname{Reshape}\left(\mathcal{H} \times_{\mathbb{A}_{l}} \mathbf{D}, \mathbb{A}_{1}, \ldots, \mathbb{A}_{L}\right) \\
& =\operatorname{Reshape}\left(\mathcal{H}, \mathbb{A}_{1}, \ldots, \mathbb{A}_{L}\right) \times_{l} \mathbf{D}
\end{aligned}
$$

Proof 3.1. The proof of Theorem 3.1 relies on the following straightforward result:

$$
\forall l \in[1, L],[\mathcal{H}]_{\mathbb{A}_{l}}=\left[\operatorname{Reshape}\left(\mathcal{H}, \mathbb{A}_{1}, \ldots, \mathbb{A}_{L}\right)\right]_{l} .
$$

This leads to $[\mathcal{B}]_{\mathbb{A}_{l}}=\left[\operatorname{Reshape}\left(\mathcal{B}, \mathbb{A}_{1}, \ldots, \mathbb{A}_{L}\right)\right]_{l}$ and $[\mathcal{H}]_{\mathbb{A}_{l}}=\left[\operatorname{Reshape}\left(\mathcal{H}, \mathbb{A}_{1}, \ldots, \mathbb{A}_{L}\right)\right]_{l}$. Applying these two results on (11), we obtain

$$
\begin{aligned}
& {\left[\operatorname{Reshape}\left(\mathcal{B}, \mathbb{A}_{1}, \ldots, \mathbb{A}_{L}\right)\right]_{l}} \\
& \quad=\mathbf{D}\left[\operatorname{Reshape}\left(\mathcal{H}, \mathbb{A}_{1}, \ldots, \mathbb{A}_{L}\right)\right]_{l} .
\end{aligned}
$$

From Equation 2, Equation 13 is equivalent to

$\operatorname{Reshape}\left(\mathcal{B}, \mathbb{A}_{1}, \ldots, \operatorname{Reshape}\left(\mathcal{H}, \mathbb{A}_{1}, \ldots, \mathbb{A}_{L}\right) \times_{l} \mathbb{A}_{L}\right) \mathbf{D}$.

Finally, one has

$$
\begin{aligned}
& \operatorname{Reshape}\left(\mathcal{H} \times \mathbb{A}_{l} \mathbf{D}, \mathbb{A}_{1}, \ldots, \mathbb{A}_{L}\right) \\
& =\operatorname{Reshape}\left(\mathcal{H}, \mathbb{A}_{1}, \ldots, \mathbb{A}_{L}\right) \times{ }_{l} \mathbf{D} .
\end{aligned}
$$

Remark Thanks to the previous proposition and the commutative property of $n$-mode product, multimode product is also commutative.

\subsection{AU-HOSVD}

With the new tools presented in the previous subsection, we are now able to introduce the AU-HOSVD. This is the purpose of the following theorem.

Theorem 3.1 (Alternative unfolding HOSVD). Let $\mathcal{H} \in \mathbb{C}^{I_{1} \times \ldots \times I_{P}}$ and $\mathbb{A}_{1} \ldots \mathbb{A}_{L}$ a partition of $\mathbb{A}$. Then, $\mathcal{H}$ may be decomposed as follows:

$$
\mathcal{H}=\mathcal{K}_{\mathbb{A}_{1} / \ldots / \mathbb{A}_{L}} \times_{\mathbb{A}_{1}} \mathbf{U}^{\left(\mathbb{A}_{1}\right)} \ldots \times_{\mathbb{A}_{L}} \mathbf{U}^{\left(\mathbb{A}_{L}\right)},
$$

where

- $\forall l \in[1, L], \mathbf{U}^{\left(\mathbb{A}_{l}\right)} \in \mathbb{C}^{\mathbb{A}_{l} \times \mathbb{A}_{l}}$ is unitary. The matrix $\mathbf{U}^{\left(\mathbb{A}_{l}\right)}$ is given by the singular value decomposition of the $\mathbb{A}_{l}$-dimension unfolding, $[\mathcal{H}]_{\mathbb{A}_{l}}=\mathbf{U}^{\left(\mathbb{A}_{l}\right)} \Sigma^{\left(\mathbb{A}_{l}\right)} \mathbf{V}^{\left(\mathbb{A}_{l}\right) H}$.

- $\mathcal{K}_{\mathbb{A}_{1} / \ldots / \mathbb{A}_{L}} \in \mathbb{C}^{I_{1} \times \ldots \times I_{P}}$ is the core tensor. It has the same properties as the HOSVD core tensor.

Notice that there are several ways to decompose a tensor with the AU-HOSVD. Each choice of the $\mathbb{A}_{1}, \ldots, \mathbb{A}_{L}$ gives a different decomposition. For a Pth-order tensor, the number of different AU-HOSVD is given by the Bell number, $B_{P}$ :

$$
\begin{aligned}
B_{1} & =1 \\
B_{P+1} & =\sum_{k=1}^{P}\left(\begin{array}{l}
P \\
k
\end{array}\right) B_{k} .
\end{aligned}
$$

The AU-HOSVD associated to the partition $\mathbb{A}_{1}, \ldots, \mathbb{A}_{L}$ is denoted AU-HOSVD $\mathbb{A}_{1}, \ldots, \mathbb{A}_{L}$. 
Proof 3.2. First, let us consider $\mathbb{A}_{1}, \ldots, \mathbb{A}_{L}$, a partition of $\mathbb{A}$. Reshape $\left(\mathcal{H}, \mathbb{A}_{1} \ldots \mathbb{A}_{L}\right)$ is a $L$ th-order tensor and may be decomposed using the HOSVD:

$$
\operatorname{Reshape}\left(\mathcal{H}, \mathbb{A}_{1}, \ldots, \mathbb{A}_{L}\right)=\mathcal{K} \times{ }_{1} \mathbf{U}^{(1)} \ldots \times_{L} \mathbf{U}^{(L)},
$$

where the matrix $\mathbf{U}^{(l)}$ is given by the singular value decomposition of the $l$-dimension unfolding, $\left[\operatorname{Reshape}\left(\mathcal{H}, \mathbb{A}_{1}, \ldots, \mathbb{A}_{L}\right)\right]_{l}=[\mathcal{H}]_{\mathbb{A}_{l}}=\mathbf{U}^{(l)} \Sigma^{(l)} \mathbf{V}^{(l) H}$.

Since the matrices $\mathbf{U}^{(l)}$ 's are unitary, Equation 15 is equivalent to

$$
\operatorname{Reshape}\left(\mathcal{H}, \mathbb{A}_{1}, \ldots, \mathbb{A}_{L}\right) \times{ }_{1} \mathbf{U}^{(1) H} \ldots \times_{L} \mathbf{U}^{(L) H}=\mathcal{K}
$$

Then, using proposition 3.1, the following equality is true:

$$
\begin{gathered}
\operatorname{Reshape}\left(\mathcal{H}, \mathbb{A}_{1}, \ldots, \mathbb{A}_{L}\right) \times{ }_{1} \mathbf{U}^{(1) H} \ldots \times_{L} \mathbf{U}^{(L) H} \\
=\operatorname{Reshape}\left(\mathcal{H} \times_{\mathbb{A}_{1}} \mathbf{U}^{(1) H} \ldots \times_{\mathbb{A}_{L}} \mathbf{U}^{(L) H},\right. \\
\left.\mathbb{A}_{1}, \ldots, \mathbb{A}_{L}\right)
\end{gathered}
$$

which leads to

$$
\operatorname{Reshape}\left(\mathcal{H} \times_{\mathbb{A}_{1}} \mathbf{U}^{(1) H} \ldots \times_{\mathbb{A}_{L}} \mathbf{U}^{(L) H}, \mathbb{A}_{1}, \ldots, \mathbb{A}_{L}\right)=\mathcal{K} .
$$

Finally, the operator Reshape $e^{-1}$ is applied

$$
\mathcal{H}=\operatorname{Reshape}^{-1}\left(\mathcal{K}, \mathbb{A}_{1}, \ldots, \mathbb{A}_{L}\right) \times_{\mathbb{A}_{1}} \mathbf{U}^{(1)} \ldots \times_{\mathbb{A}_{L}} \mathbf{U}^{(L)},
$$

which concludes the proof.

Example For a third-order tensor $\mathcal{H} \in \mathbb{C}^{I_{1} \times I_{2} \times I_{3}}$ with $\mathbb{A}_{1}=\{1,3\}, \mathbb{A}_{2}=\{2\}$, the AU-HOSVD will be written as follows:

$$
\mathcal{H}=\mathcal{K}_{\mathbb{A}_{1} / \mathbb{A}_{2}} \times_{\mathbb{A}_{1}} \mathbf{U}^{\left(\mathbb{A}_{1}\right)} \times_{\mathbb{A}_{2}} \mathbf{U}^{\left(\mathbb{A}_{2}\right)},
$$

with $\mathcal{K}_{\mathbb{A}_{1} / \mathbb{A}_{2}} \in \mathbb{C}^{I_{1} \times I_{2} \times I_{3}}, \mathbf{U}^{\left(\mathbb{A}_{1}\right)} \in \mathbb{C}^{I_{1} I_{3} \times I_{1} I_{3}}$ and $\mathbf{U}^{\left(\mathbb{A}_{2}\right)} \in$ $\mathbb{C}^{I_{2} \times I_{2}}$.

Remark Let $\mathcal{H} \in \mathbb{C}^{I_{1} \times I_{2} \ldots \times I_{P} \times I_{1} \times I_{2} \ldots \times I_{P}}$ be a $2 P$ thorder Hermitian tensor. We consider $2 L$ subsets of $\left\{I_{1}, \ldots, I_{P}, I_{1}, \ldots, I_{P}\right\}$ such as

- $\mathbb{A}_{1}, \ldots, \mathbb{A}_{L}$ and $\mathbb{A}_{L+1}, \ldots, \mathbb{A}_{2 L}$ are two partitions of $\left\{I_{1}, \ldots, I_{P}\right\}$

- $\forall l \in[1, L], \mathbb{A}_{l}=\mathbb{A}_{l+L}$

Under these conditions, the AU-HOSVD of $\mathcal{H}$ is written:

$$
\begin{aligned}
\mathcal{H}= & \mathcal{K}_{\mathbb{A}_{1} / \ldots / \mathbb{A}_{2 L}} \times \times_{\mathbb{A}_{1}} \mathbf{U}^{\left(\mathbb{A}_{1}\right)} \ldots \times_{\mathbb{A}_{L}} \mathbf{U}^{\left(\mathbb{A}_{L}\right)} \\
& \times_{\mathbb{A}_{L+1}} \mathbf{U}^{\left(\mathbb{A}_{1}\right) *} \ldots \times_{\mathbb{A}_{2 L}} \mathbf{U}^{\left(\mathbb{A}_{L}\right) *} .
\end{aligned}
$$

As discussed previously, the main motivation for introducing the new AU-HOSVD is to extract the correlated information when processing the low-rank decomposition. This is the purpose of the following proposition.

Proposition 3.2 (Low-rank approximation). Let $\mathcal{H}$, $\mathcal{H}_{c}, \mathcal{H}_{0}$ be three Pth-order tensors such that

$$
\mathcal{H}=\mathcal{H}_{c}+\mathcal{H}_{0},
$$

where $\mathcal{H}_{c}$ is a $\left(r_{\mathbb{A}_{1}}, \ldots, r_{\mathbb{A}_{L}}\right)$ low-rank tensor $^{\mathrm{c}}$ $\left(r_{\mathbb{A}_{l}}=\operatorname{rank}\left(\left[\mathcal{H}_{c}\right]_{\mathbb{A}_{l}}\right)\right)$. Then, $\mathcal{H}_{0}$ is approximated by

$$
\mathcal{H}_{0} \approx \mathcal{H} \times_{\mathbb{A}_{1}} \mathbf{U}_{0}^{\left(\mathbb{A}_{1}\right)} \mathbf{U}_{0}^{\left(\mathbb{A}_{1}\right) H} \ldots \times_{\mathbb{A}_{L}} \mathbf{U}_{0}^{\left(\mathbb{A}_{L}\right)} \mathbf{U}_{0}^{\left(\mathbb{A}_{L}\right) H}
$$

where $\mathbf{U}_{0}^{\left(\mathbb{A}_{1}\right)}, \ldots, \mathbf{U}_{0}^{\left(\mathbb{A}_{L}\right)}$ minimize the following criterion:

$$
\begin{aligned}
\left(\mathbf{U}_{0}^{\left(\mathbb{A}_{1}\right)}, \ldots, \mathbf{U}_{0}^{\left(\mathbb{A}_{L}\right)}\right) & \\
= & \operatorname{argmin} \| \mathcal{H}_{0}-\mathcal{H} \times \times_{\mathbb{A}_{1}} \mathbf{U}_{0}^{\left(\mathbb{A}_{1}\right)} \mathbf{U}_{0}^{\left(\mathbb{A}_{1}\right) H} \ldots \\
& \times{ }_{\mathbb{A}_{L}} \mathbf{U}_{0}^{\left(\mathbb{A}_{L}\right)} \mathbf{U}_{0}^{\left(\mathbb{A}_{L}\right) H} \|^{2} .
\end{aligned}
$$

In this paper, the matrices $\mathbf{U}_{0}^{\left(\mathbb{A}_{l}\right) \text { 's }}$ are given by truncation of the matrices $U^{\left(\mathbb{A}_{l}\right)}$ 's obtained by the AU-HOSVD $\mathbb{A}_{1}, \ldots, \mathbb{A}_{L}$ of $\mathcal{R}: \mathbf{U}_{0}^{\left(\mathbb{A}_{l}\right)}=\left[\mathbf{u}_{r_{\mathbb{A}_{l}}+1}^{\left(\mathbb{A}_{l}\right)} \ldots \mathbf{u}_{\mathbb{A}_{l}}^{\left(\mathbb{A}_{l}\right)}\right]$. This solution is not optimal in the sense of least squares but is easy to implement. However, thanks to the strong link with HOSVD, it should be a correct approximation. That is why iterative algorithms for AU-HOSVD will not be investigated in this paper.

Proof 3.3. By applying Reshape to Equation 21, one obtains

$$
\begin{aligned}
\operatorname{Reshape}\left(\mathcal{H}, \mathbb{A}_{1}, \ldots, \mathbb{A}_{L}\right) \\
=\operatorname{Reshape}\left(\mathcal{H}_{c}, \mathbb{A}_{1}, \ldots, \mathbb{A}_{L}\right) \\
\quad+\operatorname{Reshape}\left(\mathcal{H}_{0}, \mathbb{A}_{1}, \ldots, \mathbb{A}_{L}\right) .
\end{aligned}
$$

Then, Reshape $\left(\mathcal{H}_{c}, \mathbb{A}_{1}, \ldots, \mathbb{A}_{L}\right)$ is a $\left(r_{\mathbb{A}_{1}}, \ldots\right.$, $r_{\mathbb{A}_{L}}$ ) low-rank tensor (where $r_{\mathbb{A}_{l}}=\operatorname{rank}([$ Reshape $\left.\left.\left.\left(\mathcal{H}_{c}, \mathbb{A}_{1}, \ldots, \mathbb{A}_{L}\right)\right]_{l}\right)\right)$. Proposition 2.1 can now be applied, and this leads to

$$
\begin{aligned}
\operatorname{Reshape}( & \left.\mathcal{H}_{0}, \mathbb{A}_{1}, \ldots, \mathbb{A}_{L}\right) \\
\approx & \operatorname{Reshape}\left(\mathcal{H}, \mathbb{A}_{1}, \ldots, \mathbb{A}_{L}\right) \\
& \times{ }_{1} \mathbf{U}_{0}^{\left(\mathbb{A}_{1}\right)} \mathbf{U}_{0}^{\left(\mathbb{A}_{1}\right) H} \ldots \times{ }_{L} \mathbf{U}_{0}^{\left(\mathbb{A}_{L}\right)} \mathbf{U}_{0}^{\left(\mathbb{A}_{L}\right) H} .
\end{aligned}
$$

Finally, applying Reshape ${ }^{-1}$ to the previous equation leads to the end of the proof:

$$
\mathcal{H}_{0} \approx \mathcal{H} \times \times_{\mathbb{A}_{1}} \mathbf{U}_{0}^{\left(\mathbb{A}_{1}\right)} \mathbf{U}_{0}^{\left(\mathbb{A}_{1}\right) H} \ldots \times_{\mathbb{A}_{L}} \mathbf{U}_{0}^{\left(\mathbb{A}_{L}\right)} \mathbf{U}_{0}^{\left(\mathbb{A}_{L}\right) H} .
$$

Discussion on choice of partition and complexity As mentioned previously, the total number of AU-HOSVD for a $P$ th-order tensor is equal to $B_{P}$. Since this number could become significant, it is important to have a procedure to find good partitions for the AU-HOSVD computation. We propose a two-step procedure. Since the AU-HOSVD has been developed for LR reduction, 
the most important criterion is to choose the partitions which emphasize deficient ranks. For some applications, it is possible to use a priori knowledge to select some partitions as will be shown in Section 5 for polarimetric STAP. Next, another step is needed if several partitions induce an AU-HOSVD with a deficient rank. At this point, we propose to maximize a criterion (see Section 5.3 for examples) over the remaining partitions.

Concerning the complexity, the number of operation necessary to compute the HOSVD of a Pth-order tensor is equal to $4\left(\prod_{p} I_{p}\right)\left(\sum_{p} I_{p}\right)$ [3]. Similarly, the complexity of the AU-HOSVD is equal to $4\left(\prod_{p} I_{p}\right)\left(\sum_{l} I_{\mathbb{A}_{l}}\right)$.

\section{Low-rank filter and detector for multidimensional data based on the alternative unfolding HOSVD}

We propose in this section to apply this new decomposition to derive a tensorial LR filter and a tensorial LR detector for multidimensional data. We consider the case of a $P$-dimensional data $X$ composed of a target described by its steering tensor $\mathcal{S}$ and two additive noises: $\mathcal{N}$ and $\mathcal{C}$. We assume that we have $K$ secondary data $X_{k}$ containing only the additive noises. This configuration could be summarized as follows:

$$
\begin{aligned}
\boldsymbol{X} & =\alpha \mathcal{S}+\mathcal{C}+\mathcal{N} \\
x_{k} & =\mathcal{C}_{k}+\mathcal{N}_{k} \quad k \in[1, K] .
\end{aligned}
$$

where $\mathcal{X}, \boldsymbol{x}_{k}, \mathcal{C}, \mathcal{C}_{k}, \mathcal{N}, \mathcal{N}_{k} \in \mathbb{C}^{I_{1} \times \ldots \times I_{P}}$. We assume that $\mathcal{N}, \mathcal{N}_{k} \sim \mathcal{C N}\left(\mathbf{0}, \sigma^{2} S q M a t^{-1}\left(\mathbf{I}_{I_{1} \ldots I_{p}}\right)\right)$ and $\mathcal{C}, \mathcal{C}_{k} \sim \mathcal{C N}\left(\mathbf{0}, \mathcal{R}_{c}\right)$ $\left(\right.$ SqMat $\left.^{-1}\left(\mathbf{I}_{I_{1} \ldots I_{p}}\right), \mathcal{R}_{c} \in \mathbb{C}^{I_{1} \times \ldots \times I_{P} \times I_{1} \times \ldots \times I_{P}}\right)$. These notations mean $\operatorname{vec}(\mathcal{N}), \operatorname{vec}\left(\mathcal{N}_{k}\right) \sim \mathcal{C N}\left(\mathbf{0}, \sigma^{2} \mathbf{I}_{I_{1} \ldots I_{p}}\right)$ and $\operatorname{vec}(\mathcal{C})$, $\operatorname{vec}\left(\mathfrak{C}_{k}\right) \sim \mathcal{C N}\left(\mathbf{0}, \operatorname{SqMat}\left(\mathcal{R}_{c}\right)\right)$. We denote $\mathcal{R}=\mathcal{R}_{c}+$ $\sigma^{2} S_{q M a t}{ }^{-1}\left(\mathbf{I}_{I_{1} \ldots I_{p}}\right)$ the covariance tensor of the total interference. We assume in the following that the additive noise $\mathcal{C}$ (and hence also $\mathcal{C}_{k}$ ) has a low-rank structure.

\subsection{LR filters}

Proposition 4.1 (Optimal tensor filter). The optimal tensor filter, $\mathcal{W}$, is defined as the filter which maximizes the SINR output

$$
\operatorname{SINR}_{\text {out }}=\frac{|<\mathcal{W}, \mathfrak{S}>|^{2}}{\operatorname{vec}(\mathcal{W})^{H} \operatorname{SqMat}(\mathcal{R}) \operatorname{vec}(\mathcal{W})} .
$$

It is given by the following expression:

$$
\mathcal{W}_{\text {opt }}=\operatorname{vec}^{-1}\left(\operatorname{SqMat}(\mathcal{R})^{-1} \operatorname{vec}(\mathcal{S})\right) \text {. }
$$

Proof 4.1. See Appendix 1.

In practical cases, $\mathcal{R}$ is unknown. Hence, we propose an adaptive version:

$$
\hat{\mathcal{W}}_{\text {opt }}=\operatorname{vec}^{-1}\left(\operatorname{SqMat}(\hat{\mathcal{R}})^{-1} \operatorname{vec}(\boldsymbol{S})\right),
$$

where $\hat{\mathcal{R}}$ is the estimation of $\mathcal{R}$ given by the SCT from Equation 9. This filter is equivalent to the vector filter. In order to reach correct performance [29], $K=$ $2 I_{1} \ldots I_{P}$ secondary data are necessary. As with the vectorial approach, it is interesting to use the low-rank structure of $\mathcal{C}$ to reduce this number $K$.

Proposition 4.2 (Low-rank tensor filter). Let $\mathbb{A}_{1}, \ldots, \mathbb{A}_{L}$ be a partition of $\{1, \ldots, P\}$. The low-rank tensor filter associated to AU-HOSVD $\mathbb{A}_{1}, \ldots, \mathbb{A}_{L}$, which removes the low-rank noise $\mathbf{C}$, is given by

$$
\begin{aligned}
\mathcal{W}_{l r\left(\mathbb{A}_{1}, \ldots, \mathbb{A}_{P}\right)}= & \mathcal{S} \times_{\mathbb{A}_{1}} \mathbf{U}_{0}^{\left(\mathbb{A}_{1}\right)} \mathbf{U}_{0}^{\left(\mathbb{A}_{1}\right) H} \ldots \\
& \times \mathbb{A}_{L} \mathbf{U}_{0}^{\left(\mathbb{A}_{L}\right)} \mathbf{U}_{0}^{\left(\mathbb{A}_{L}\right) H} \\
y= & \left|<\mathcal{W}_{\mathbb{A}_{1}, \ldots, \mathbb{A}_{P}}, \boldsymbol{X}>\right| .
\end{aligned}
$$

The matrices $\mathrm{U}_{0}^{\left(\mathbb{A}_{l}\right)}$ 's are given by truncation of the matrices $\mathbf{U}^{\left(\mathbb{A}_{l}\right)}$ 's obtained by the AU-HOSVD $\mathbb{A}_{\mathbb{A}_{1}, \ldots, \mathbb{A}_{L}}$ of $\mathcal{R}: \mathbf{U}_{0}^{\left(\mathbb{A}_{l}\right)}=$ $\left[\mathbf{u}_{r_{\mathbb{A}_{l}}+1}^{\left(\mathbb{A}_{l}\right)} \ldots \mathbf{u}_{\mathbb{A}_{l}}^{\left(\mathbb{A}_{l}\right)}\right]$.

For a $P$-dimensional configuration, $B_{P}$ filters will be obtained.

\section{Proof 4.2. See Appendix 2.}

In its adaptive version, denoted $\hat{\mathcal{W}}_{\operatorname{lr}\left(\mathbb{A}_{1} / \ldots / \mathbb{A}_{L}\right)}$, the matrices $\mathbf{U}_{0}^{\left(\mathbb{A}_{1}\right)}, \ldots, \mathbf{U}_{0}^{\left(\mathbb{A}_{L}\right)}$ are replaced by their estimates $\hat{\mathbf{U}}_{0}^{\left(\mathbb{A}_{1}\right)}, \ldots, \hat{\mathbf{U}}_{0}^{\left(\mathbb{A}_{L}\right)}$.

The number of secondary data necessary to reach classical performance is not known. In the vectorial case, the performance of LR filter depends on the deficient rank $[10,11]$. It will be similar for the LR tensor filters. This implies that the choice of the partition $\mathbb{A}_{1}, \ldots, \mathbb{A}_{L}$ is critical.

\subsection{LR detectors}

In a detection point of view, the problem can also be stated as the following binary hypothesis test:

$$
\left\{\begin{array}{l}
H_{0}: \mathcal{X}=\mathcal{C}+\mathcal{N}, \boldsymbol{X}_{k}=\mathcal{C}_{k}+\mathcal{N}_{k}, k \in[1, K] \\
H_{1}: \mathcal{X}=\alpha \mathcal{S}+\mathcal{C}+\mathcal{N}, \boldsymbol{X}_{k}=\mathcal{C}_{k}+\mathcal{N}_{k}, k \in[1, K]
\end{array}\right.
$$

Table 1 Description of the LR filters and LR detectors provided by AU-HOSVD for polarimetric STAP

\begin{tabular}{lcccc}
\hline Partition & Filters & Detectors & Ranks & Methods \\
\hline $\mathbb{A}_{1}=\{1,2,3\}$ & $\hat{\mathcal{W}}_{\operatorname{lr}(1,2,3)}$ & $\hat{\Lambda}_{(1,2,3)}$ & $r_{123}$ & Vector \\
$\mathbb{A}_{1}=\{1\}, \mathbb{A}_{2}=\{2\}, \mathbb{A}_{3}=\{3\}$ & $\hat{\mathcal{W}}_{\operatorname{lr}(1 / 2 / 3)}$ & $\hat{\Lambda}_{(1 / 2 / 3)}$ & $r_{1}, r_{2}, r_{3}$ & HOSVD \\
$\mathbb{A}_{1}=\{1,2\}, \mathbb{A}_{2}=\{3\}$ & $\hat{\mathcal{W}}_{\operatorname{lr}(1,2 / 3)}$ & $\hat{\Lambda}_{(1,2 / 3)}$ & $r_{12}, r_{3}$ & AU-HOSVD \\
$\mathbb{A}_{1}=\{1\}, \mathbb{A}_{2}=\{2,3\}$ & $\hat{\mathcal{W}}_{\operatorname{lr}(1 / 2,3)}$ & $\hat{\Lambda}_{(1 / 2,3)}$ & $r_{1}, r_{23}$ & AU-HOSVD \\
$\mathbb{A}_{1}=\{1,3\}, \mathbb{A}_{2}=\{2\}$ & $\hat{\mathcal{W}}_{\operatorname{lr}(1,3 / 2)}$ & $\hat{\Lambda}_{(1,3 / 2)}$ & $r_{13}, r_{2}$ & AU-HOSVD \\
\hline
\end{tabular}


Table 2 Summary of the value of the ranks for the two scenarios: $\rho=1, \rho=0.5$

\begin{tabular}{lccccccc}
\hline & $\boldsymbol{r}_{\mathbf{1}}$ & $\boldsymbol{r}_{\mathbf{2}}$ & $\boldsymbol{r}_{\mathbf{3}}$ & $\boldsymbol{r}_{\mathbf{1 2}}$ & $\boldsymbol{r}_{\mathbf{2 3}}$ & $\boldsymbol{r}_{\mathbf{1 3}}$ & $\boldsymbol{r}_{\mathbf{1 2 3}}$ \\
\hline$\rho=1$ & Full & Full & 2 & 15 & 16 & 16 & 30 \\
$\rho=0.5$ & Full & Full & Full & 15 & Full & Full & 45 \\
\hline
\end{tabular}

Proposition 4.3 (Low-rank tensor detector). Let $\mathbb{A}_{1}, \ldots, \mathbb{A}_{L}$ be a partition of $\{1, \ldots, P\}$. The low-rank tensor detector associated to $A U-H O S V D_{\mathbb{A}_{1}, \ldots, \mathbb{A}_{L}}$, which removes the low-rank noise $\mathcal{C}$ and performs the generalized likelihood ratio test (GLRT), is given by

$$
\Lambda_{\mathbb{A}_{1} \ldots \mathbb{A}_{L}}=\frac{\left|<\mathcal{S}_{\mathbb{A}_{1} \ldots \mathbb{A}_{L}}, \boldsymbol{X}_{\mathbb{A}_{1} \ldots \mathbb{A}_{L}}>\right|^{2}}{<\mathcal{S}_{\mathbb{A}_{1} \ldots \mathbb{A}_{L}}, \mathcal{S}_{\mathbb{A}_{1} \ldots \mathbb{A}_{L}}><X_{\mathbb{A}_{1} \ldots \mathbb{A}_{L}}, \boldsymbol{X}_{\mathbb{A}_{1} \ldots \mathbb{A}_{L}}>}
$$

where

$$
\begin{aligned}
x_{\mathbb{A}_{1} \ldots \mathbb{A}_{L}} & =X \times_{\mathbb{A}_{1}} \mathbf{U}_{0}^{\left(\mathbb{A}_{1}\right)} \mathbf{U}_{0}^{\left(\mathbb{A}_{1}\right) H} \ldots \times_{\mathbb{A}_{L}} \mathbf{U}_{0}^{\left(\mathbb{A}_{L}\right)} \mathbf{U}_{0}^{\left(\mathbb{A}_{L}\right) H} \\
\mathcal{S}_{\mathbb{A}_{1} \ldots \mathbb{A}_{L}} & =\mathcal{S} \times_{\mathbb{A}_{1}} \mathbf{U}_{0}^{\left(\mathbb{A}_{1}\right)} \mathbf{U}_{0}^{\left(\mathbb{A}_{1}\right) H} \ldots \times_{\mathbb{A}_{L}} \mathbf{U}_{0}^{\left(\mathbb{A}_{L}\right)} \mathbf{U}_{0}^{\left(\mathbb{A}_{L}\right) H}
\end{aligned}
$$

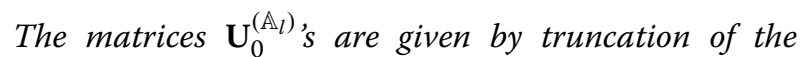
matrices $\mathbf{U}^{\left(\mathbb{A}_{l}\right)}$ 's obtained by the AU-HOSVD $D_{\mathbb{A}_{1}, \ldots, \mathbb{A}_{L}}$ of $\mathcal{R}: \mathbf{U}_{0}^{\left(\mathbb{A}_{l}\right)}=\left[\mathbf{u}_{r_{\mathbb{A}_{l}}+1}^{\left(\mathbb{A}_{l}\right)} \ldots \mathbf{u}_{\mathbb{A}_{l}}^{\left(\mathbb{A}_{l}\right)}\right]$.

\section{Proof 4.3. See Appendix 3.}

In its adaptive version, denoted as $\hat{\Lambda}_{\mathbb{A}_{1} \ldots \mathbb{A}_{L}}$, the matrices $\mathbf{U}_{0}^{\left(\mathbb{A}_{1}\right)}, \ldots, \mathbf{U}_{0}^{\left(\mathbb{A}_{L}\right)}$ are replaced by their estimates $\hat{\mathbf{U}}_{0}^{\left(\mathbb{A}_{1}\right)}, \ldots, \hat{\mathbf{U}}_{0}^{\left(\mathbb{A}_{L}\right)}$.

\subsection{Particular case}

When the partition $\mathbb{A}_{1}=\{1, \ldots, P\}$ is chosen, the filter and the detector obtained by the AU-HOSVD are equal to the vectorial one. In other words, it is equivalent to apply the operator vec on Equations 24 and 25 and use the vectorial method. We denote $m=I_{1} \ldots I_{P}, \mathbf{x}=\operatorname{vec}(\boldsymbol{X})$ and $\mathbf{s}=\operatorname{vec}(\mathcal{S})$. We obtain the basis of the orthogonal clutter subspace $\mathbf{U}_{0}$ by taking the last $(m-r)$ columns of $\mathbf{U}$ which is computed by the SVD of $\operatorname{SqMat}(\mathcal{R})=\mathbf{U} \Sigma \mathbf{V}^{H}$. From this basis, the low-rank filter is then equal to $[10,11]$ :

$$
\begin{aligned}
\mathbf{w}_{l r} & =\mathbf{U}_{0} \mathbf{U}_{0}^{H} \mathbf{s}, \\
y_{l r} & =\left|<\mathbf{w}_{l r}, \mathbf{x}>\right| .
\end{aligned}
$$

In its adaptive version, denoted $\hat{\mathbf{w}}_{l r}$, the matrix $\mathbf{U}_{0}$ is replaced by its estimate $\hat{\mathbf{U}}_{0}$.

Similarly, the detector is equal to the low-rank normalized matched filter proposed in [30,31]:

$$
\Lambda_{\mathrm{LR}-\mathrm{NMF}}=\frac{\left|\mathbf{s}^{H} \mathbf{U}_{0} \mathbf{U}_{0}^{H} \mathbf{x}\right|^{2}}{\left(\mathbf{s}^{H} \mathbf{U}_{0} \mathbf{U}_{0}^{H} \mathbf{s}\right)\left(\mathbf{x}^{H} \mathbf{U}_{0} \mathbf{U}_{0}^{H} \mathbf{x}\right)} .
$$

In its adaptive version, denoted $\hat{\Lambda}_{\mathrm{LR}-\mathrm{NMF}}$, the matrix $\mathbf{U}_{0}$ is replaced by its estimate $\hat{\mathbf{U}}_{0}$.

\section{Application to polarimetric STAP \\ 5.1 Model}

We propose to apply the LR filters and the LR detectors derived in the previous section to polarimetric STAP. STAP is applied to airborne radar in order to detect moving targets [20]. Typically, the radar receiver consists of an array of $N$ antenna elements processing $M$ pulses in a coherent processing interval. In polarimetric configuration, each element transmits and receives in both $\mathrm{H}$ and $\mathrm{V}$ polarizations, resulting in three polarimetric channels $(\mathrm{HH}, \mathrm{VV}, \mathrm{HV} / \mathrm{VH})$. The number of dimensions of polarimetric STAP data is then equal to three: $N$ antenna, $M$ pulses, and three polarimetric channels.

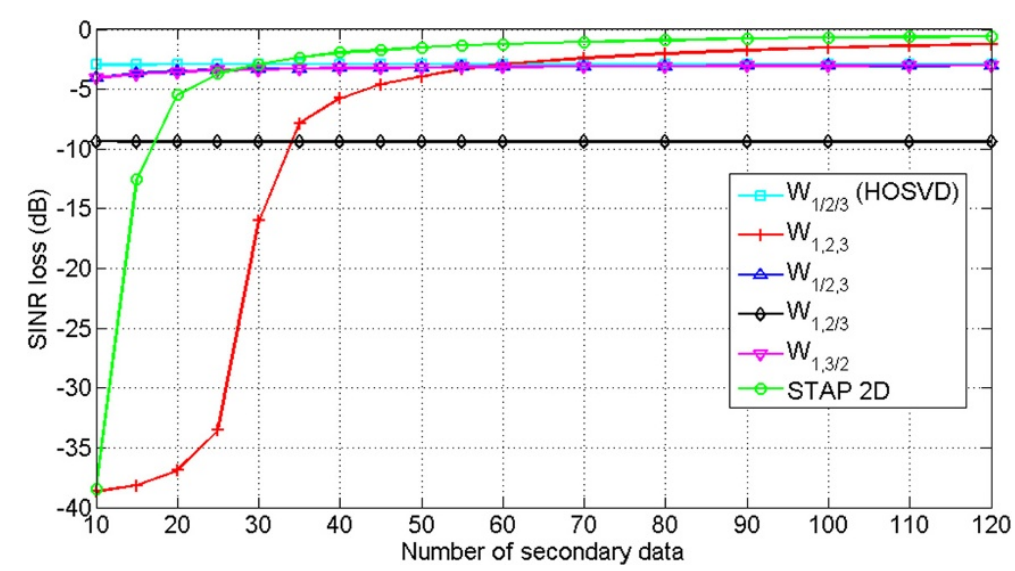

Figure 1 SINR losses versus $K$ for $\rho=\mathbf{1}, \mathbf{C N R}=\mathbf{4 0} \mathbf{d B}$. Target located at position $\left(\theta=0^{\circ}, v=10 \mathrm{~m} \mathrm{~s}^{-1}\right)$. 


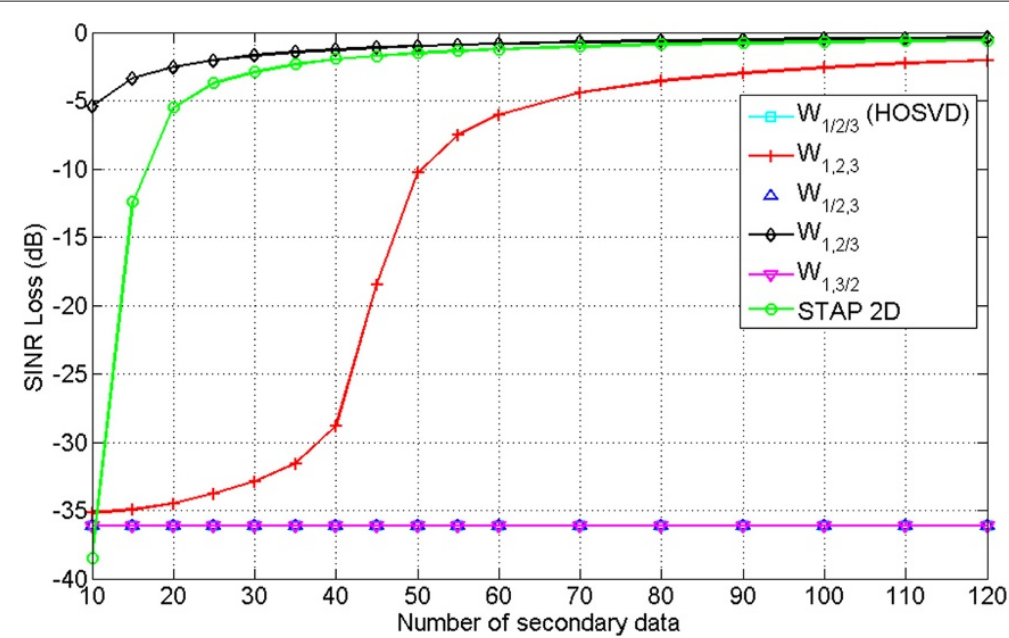

Figure 2 SINR losses versus $\boldsymbol{K}$ for $\boldsymbol{\rho}=\mathbf{0 . 5}, \mathbf{C N R}=\mathbf{4 0} \mathrm{dB}$. Target located at position $\left(\theta=0^{\circ}, v=10 \mathrm{~m} \mathrm{~s}^{-1}\right)$.

We are in the data configuration proposed in Equations 24 and 25 which is recalled in the following equations:

$$
\begin{aligned}
X & =\alpha \mathcal{S}+\mathcal{C}+\mathcal{N} \\
x_{k} & =\mathcal{C}_{k}+\mathcal{N}_{k} \quad k \in[1, K],
\end{aligned}
$$

where $\boldsymbol{X}, \boldsymbol{x}_{k} \in \mathbb{C}^{M \times N \times 3}$. The steering tensor $\mathcal{S}$ and the responses of the background $\mathcal{C}$ and $\mathcal{C}_{k}$, called clutter in STAP, are obtained from the model proposed in [22]. $\mathcal{N}$ and $\mathcal{N}_{k}$, which arise from the electrical components of the radar, are distributed as a white Gaussian noise.

The steering tensor, $\mathcal{S}$, is formed as follows:

$$
\mathcal{S}(\theta, v)=v e c^{-1}\left(\begin{array}{c}
\mathbf{s}_{\mathrm{HH}}(\theta, v) \\
\alpha_{\mathrm{VV}} \mathbf{s}_{\mathrm{HH}}(\theta, v) \\
\alpha_{\mathrm{VH}} \mathbf{s}_{\mathrm{HH}}(\theta, v)
\end{array}\right),
$$

where $\mathbf{s}_{\mathrm{HH}}(\theta, v)$ is the $2 \mathrm{D}$ steering vector [20], characterized by the angle of arrival (AOA) $\theta$ and the speed $v$ of the target. $\alpha_{\mathrm{VV}}$ and $\alpha_{\mathrm{VH}}$ are two complex coefficients. These coefficients are assumed to be known. This is the case when the detection process concerns a particular target (surface, double-bounds, volume, ...). The covariance tensor, denoted as $\mathcal{R} \in \mathbb{C}^{M \times N \times 3 \times M \times N \times 3}$, of the two noises $\left(\mathcal{C}+\mathcal{N}\right.$ and $\left.\mathcal{C}_{k}+\mathcal{N}_{k}\right)$ is given by

$$
\mathcal{R}=\operatorname{SqMat}^{-1}\left(\mathbf{R}_{p c}+\sigma^{2} \mathbf{I}_{3 M N}\right),
$$

where $\sigma^{2}$ is the power of the white noise. $\mathbf{R}_{p c}$ is built as follows:

$$
\mathbf{R}_{p c}=\left(\begin{array}{ccc}
\mathbf{R}_{\mathbf{c}} & \rho \sqrt{\gamma_{V V}} \mathbf{R}_{\mathbf{c}} & \mathbf{0} \\
\rho^{*} \sqrt{\gamma_{\mathrm{VV}}} \mathbf{R}_{\mathbf{c}} & \gamma_{\mathrm{VV}} \mathbf{R}_{\mathbf{c}} & \mathbf{0} \\
\mathbf{0} & \mathbf{0} & \gamma_{\mathrm{VH}} \mathbf{R}_{\mathbf{c}}
\end{array}\right),
$$

where $\mathbf{R}_{\mathbf{c}} \in \mathbb{C}^{M N \times M N}$ is the covariance matrix of the $\mathrm{HH}$ channel clutter, built as the $2 \mathrm{D}$ clutter, which is known to have a LR structure [20]. $\gamma_{\mathrm{VV}}$ and $\gamma_{\mathrm{VH}}$ are two coefficients relative to the nature of the ground, and $\rho$ is the correlation coefficient between the channels HH and VV. Due to the structure of $\mathbf{R}_{p c}$, the low-rank structure of the clutter is preserved.

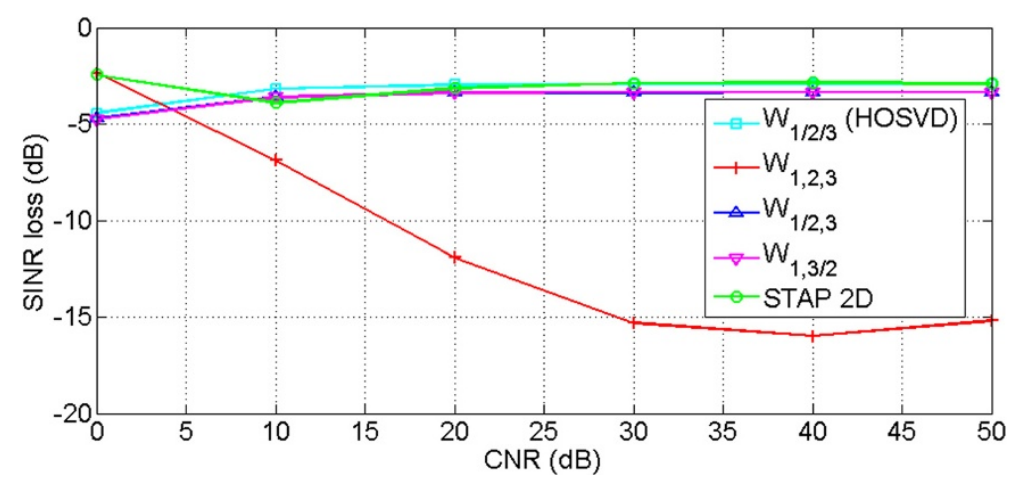

Figure 3 SINR losses versus CNR for $\boldsymbol{\rho}=\mathbf{1}, \boldsymbol{K}=\mathbf{3 0}, \boldsymbol{N}_{\text {rea }}=\mathbf{1}, \mathbf{0 0 0}$. Target located at $\left(\theta=0^{\circ}, v=10 \mathrm{~m} \mathrm{~s}^{-1}\right)$. 


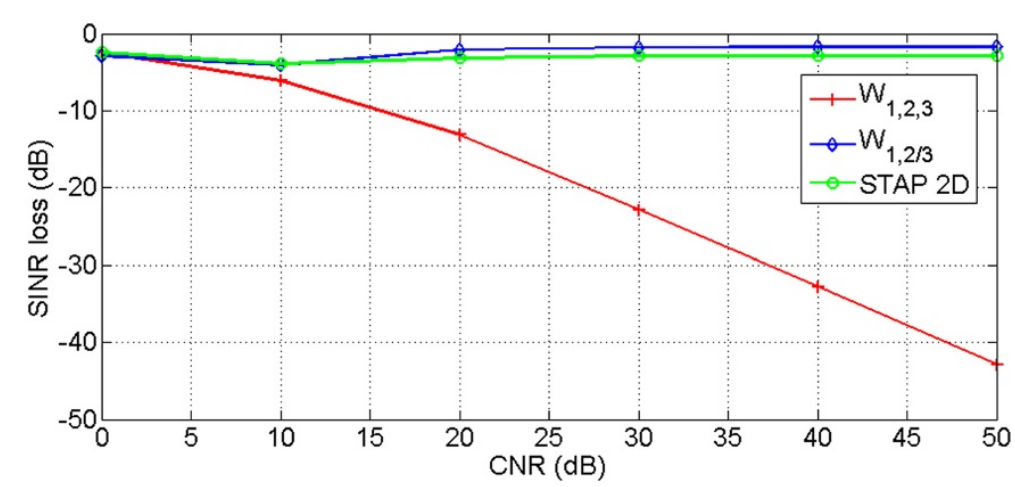

Figure 4 SINR losses versus CNR for $\boldsymbol{\rho}=\mathbf{0 . 5}, \boldsymbol{K}=\mathbf{3 0}, \boldsymbol{N}_{\text {rea }}=\mathbf{1}, \mathbf{0 0 0}$. Target located at $\left(\theta=0^{\circ}, \mathrm{v}=10 \mathrm{~m} \mathrm{~s}^{-1}\right)$.

In the following subsection, we discuss about the choice of partitions in this particular context.

\subsection{Choice of partition $\mathbb{A}$}

For polarimetric STAP, we have $P=3$ and $\mathbb{A}=\{1,2,3\}$ : $B_{3}=5$ LR filters and LR detectors are obtained. The different choices of partition are presented in Table 1. All filters and detectors are computed with the AU-HOSVD. Nevertheless, the first two partitions are particular cases. When $\mathbb{A}_{1}=\{1,2,3\}$, the algorithms are equal to the vectorial one as mentioned in Section 4.3. When $\mathbb{A}_{1}=\{1\}$, $\mathbb{A}_{2}=\{2\}, \mathbb{A}_{3}=\{3\}$, we obtain the same LR filter and LR detector as those given by the HOSVD. The ranks relative to the LR filters and LR detectors are described in the following:

- The rank $r_{1}$ is the spatial rank, and the rank $r_{2}$ is the temporal rank. They depend on radar parameters, and in most cases, they are not deficient.

- $r_{3}$ could be deficient depending on the nature of the data and especially on the correlation coefficient $\rho$ between the polarimetric channels.
- $r_{12}$ is the same as the $2 \mathrm{D}$ low-rank vector case and can be calculated by the Brennan's rule [21].

- $r_{123}$ is deficient and is linked to $r_{3}$ and $r_{12}$.

- $r_{13}$ and $r_{23}$ could be deficient and depends on $r_{1}, r_{2}$ and $r_{3}$.

\subsection{Performance criteria}

In order to evaluate the performance of our LR filters, we evaluate the SINR loss defined as follows [20]:

$$
\rho_{\text {loss }}=\frac{\text { SINR }_{\text {out }}}{\text { SINR }_{\text {max }}},
$$

where SINR out $_{\text {is }}$ the SINR at the output of the LR tensor STAP filter and SINR $\max$ the SINR at the output of the optimal filter $\mathcal{W}_{\text {opt }}$. The SINR out $_{\text {is maximum when }}$ $\mathcal{W}=\mathcal{W}_{\text {opt }}=\operatorname{vec}^{-1}\left(\operatorname{SqMat}(\mathcal{R})^{-1} \operatorname{vec}(\mathcal{S})\right)$. After some developments, the SINR loss is equal to [1]

$$
\begin{aligned}
& \rho_{\text {loss }} \\
& =\frac{\left|\left(\operatorname{vec}\left(\mathcal{W}_{l r}\right)^{H} \operatorname{vec}(\boldsymbol{S})\right)\right|^{2}}{\operatorname{vec}\left(\mathcal{W}_{l r}\right)^{H} \operatorname{SqMat}(\mathcal{R}) \operatorname{vec}\left(\mathcal{W}_{l r}\right) \operatorname{vec}(\mathbf{S})^{H} \operatorname{SqMat}(\mathcal{R})^{-1} \operatorname{vec}(\mathbf{S})} .
\end{aligned}
$$

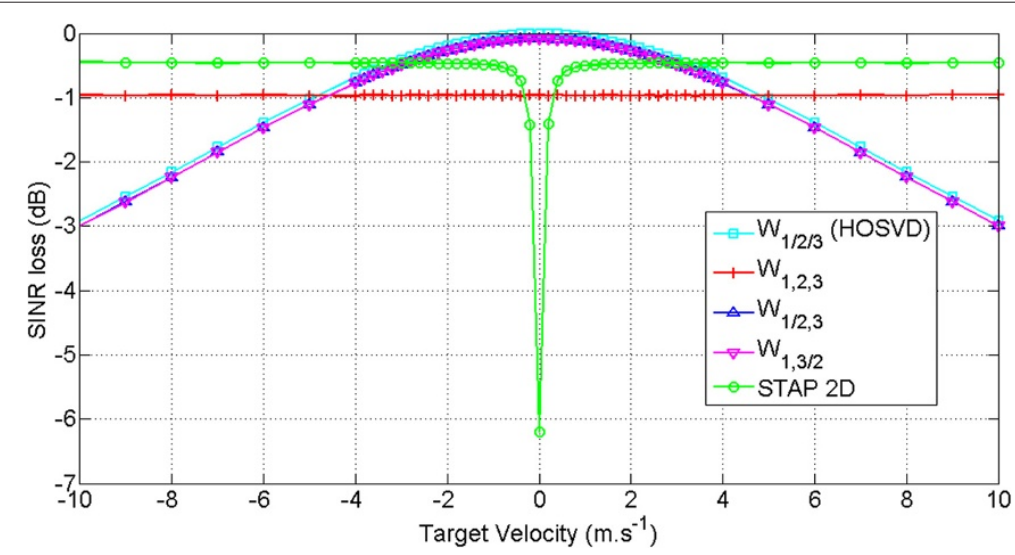

Figure 5 SINR losses versus target velocity for $\rho=1, C N R=40 \mathrm{~dB}, \boldsymbol{K}=\mathbf{1 8 0}, \boldsymbol{N}_{\text {rea }}=\mathbf{1}, \mathbf{0 0 0}$. Target located at $\theta=0^{\circ}$. 


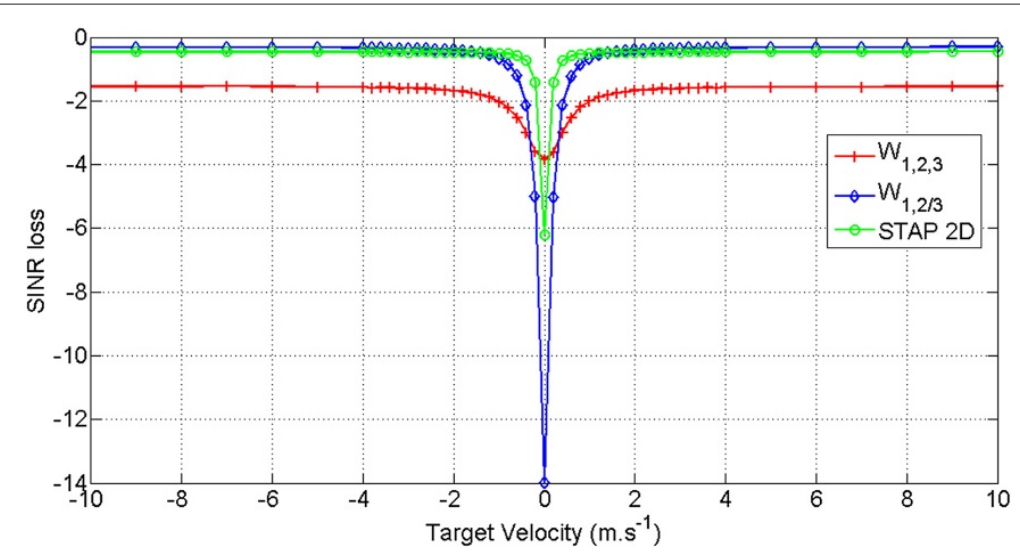

Figure 6 SINR losses versus target velocity for $\rho=0.5, C N R=40 \mathrm{~dB}, \boldsymbol{K}=\mathbf{1 8 0}, \boldsymbol{N}_{\text {rea }}=\mathbf{1}, \mathbf{0 0 0}$. Target located at $\theta=0^{\circ}$

For the moment, as the analytical formulation of the SINR loss for the tensorial approach is not available, it will be evaluated using Monte Carlo simulations.

In order to evaluate the performance of our LR detectors, we use the probability of false alarm (Pfa) and probability of detection $(\mathrm{Pd})$ :

$$
\begin{aligned}
\operatorname{Pfa} & =\operatorname{Pr}\left(\hat{\Lambda}_{\mathbf{A}_{1} \ldots \mathbf{A}_{L}}>\eta / H_{0}\right) \\
\operatorname{Pd} & =\operatorname{Pr}\left(\hat{\Lambda}_{\mathbf{A}_{1} \ldots \mathbf{A}_{L}}>\eta / H_{1}\right),
\end{aligned}
$$

where $\eta$ is the detector threshold. Since there is no analytical formulation for Pfa and Pd (for the adaptive version) even in the vectorial case, Monte Carlo simulations are used to evaluate them.

\subsection{Simulations}

Parameters The simulations are performed with the following parameters. The target is characterized by an angle of arrival (AOA) of $\theta=0^{\circ}$ and a speed of $v=10 \mathrm{~m} \mathrm{~s}^{-1}$, a case where the 2D STAP is known to be inefficient because the target is close to the clutter ridge. The radar receiver contains $N=8$ sensors processing $M=8$ pulses. The platform speed $V$, is equal to $100 \mathrm{~m} \mathrm{~s}^{-1}$. For the clutter, we consider two cases: $\rho=1$, i.e., the channels $\mathrm{HH}$ and VV are entirely correlated and $\rho=0.5$. The SNR is equal to $45 \mathrm{~dB}$ and the clutter-to-noise ratio (CNR) to $40 \mathrm{~dB} . r_{1}, r_{2}, r_{3}$, and $r_{12}$ can be calculated based on the radar configuration. $r_{13}$ depends on the value of $\rho . r_{123}$ and $r_{23}$ are estimated according to the singular values of the different unfoldings of $\mathcal{R}$. The results are presented in Table 2. All Monte-Carlo simulations are performed with $N_{\text {rea }}=1,000$ samples, except the probability of false alarm where $N_{\text {rea }}=100$.

Results on SINR losses Figures 1 and 2 show the SINR losses for each filter as a function of $K$. SINR losses are obtained from Monte-Carlo simulations using Equation 43. On both figures, the SINR loss of the

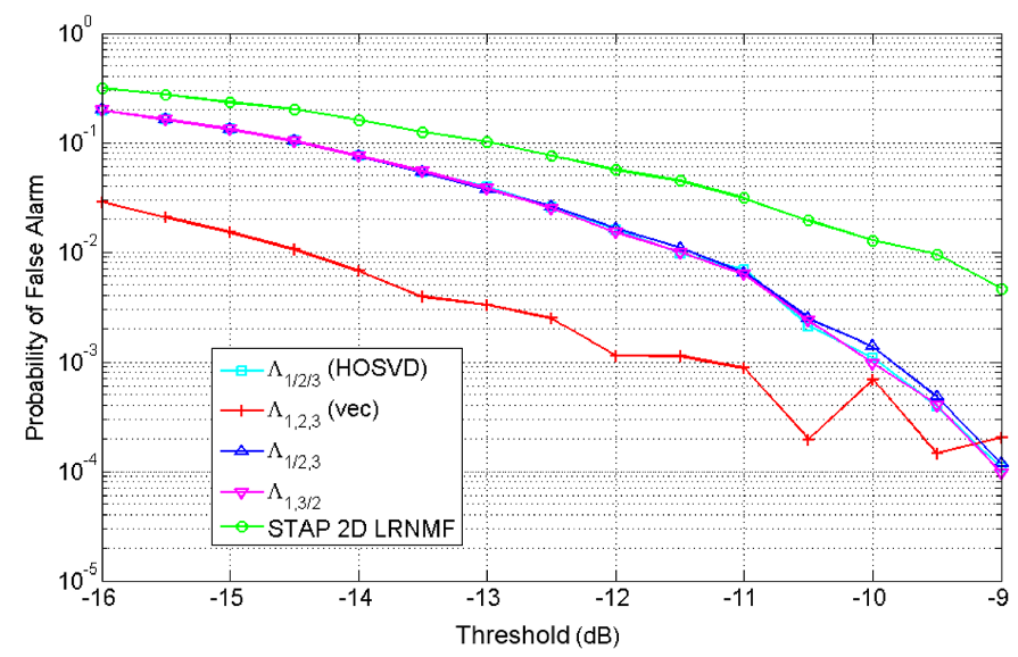

Figure 7 Pfa versus threshold for $\rho=1, K=30, \mathrm{CNR}=40 \mathrm{~dB}, N_{\text {rea }}=100$. 


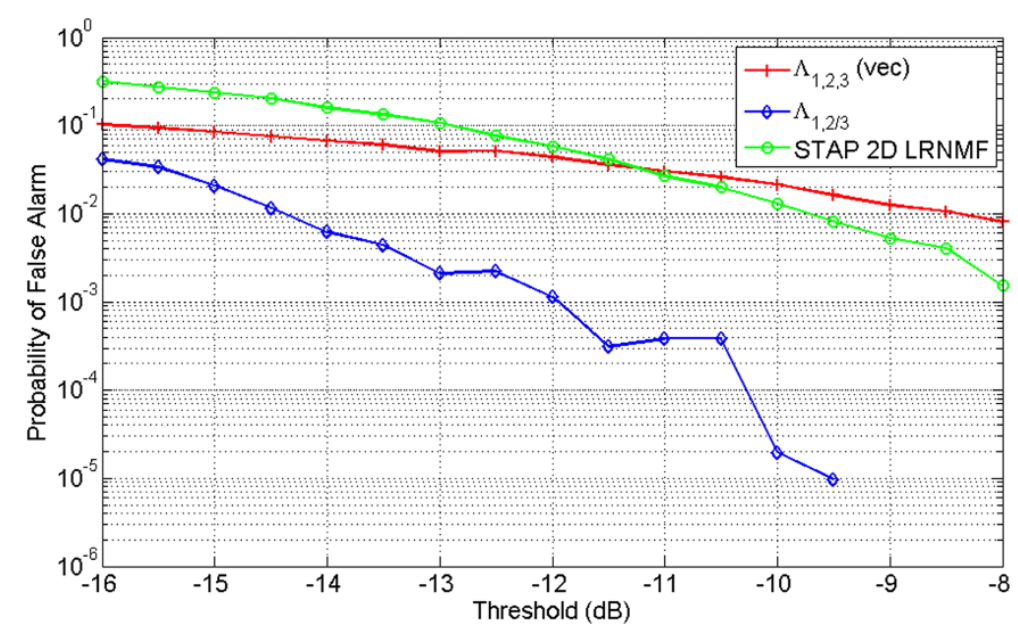

Figure 8 Pfa versus threshold for $\rho=0.5, K=30, \mathrm{CNR}=40 \mathrm{~dB}, N_{\text {rea }}=100$.

2D STAP is plotted for comparison. The well-known result is obtained: the SINR loss reaches $-3 \mathrm{~dB}$ when $K=2 r_{12}=30$, and it tends to $0 \mathrm{~dB}$ as $K$ increases. Similarly, the SINR loss of $\hat{\mathcal{W}}_{\operatorname{lr}(1,2,3)}$ reaches $-3 \mathrm{~dB}$ when $K=2 r_{123}$ (60 for $\rho=1$ and 90 for $\left.\rho=0.5\right)$. When $\rho=1$, all LR filters achieve reasonable performance since all ranks, except $r_{1}$ and $r_{2}$, are deficient. $\hat{\mathcal{W}}_{\operatorname{lr}(1 / 2 / 3)}$, $\hat{\mathcal{W}}_{\operatorname{lr}(1 / 2,3)}$, and $\hat{\mathcal{W}}_{\operatorname{lr}(1,3 / 2)}$, which can only be obtained by AU-HOSVD, outperform $\hat{\mathcal{W}}_{\operatorname{lr}(1,2,3)}$ and the 2D STAP for a small number of secondary data. This situation is more realistic since the assumption of homogeneity of the data is no longer true when $K$ is too large. $\hat{\mathcal{W}}_{\operatorname{lr}(1,2 / 3)}$ has poor performance in this scenario.

When $\rho=0.5, \hat{\mathcal{W}}_{\operatorname{lr}(1,2 / 3)}$ outperforms $\hat{\mathcal{W}}_{\operatorname{lr}(1,2,3)}$ and the 2D STAP regardless of the number of secondary data. This corresponds to a more realistic scenario, since the channels $\mathrm{HH}$ and VV are not entirely correlated. $\hat{\mathcal{W}}_{\operatorname{lr}(1 / 2 / 3)}$,

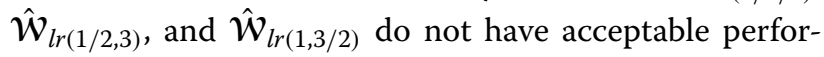
mance. This is explained by the fact that all ranks pertaining to these filters are full and no projection can be done as mentioned at the end of Section 3. These filters (for $\rho=0.5$ ) will not be studied in the rest of the simulations.

Figures 3 and 4 show the SINR loss as a function of the CNR for $K=2 r_{12}=30$ secondary data. They show that our filters are more robust than the vectorial one for polarimetric STAP configuration.

Figures 5 and 6 show the SINR loss as a function of the target velocity for $K=180$. For both cases, the 2D STAP achieves the expected performance. For $\rho=1$, the difference in polarimetric properties between the target and

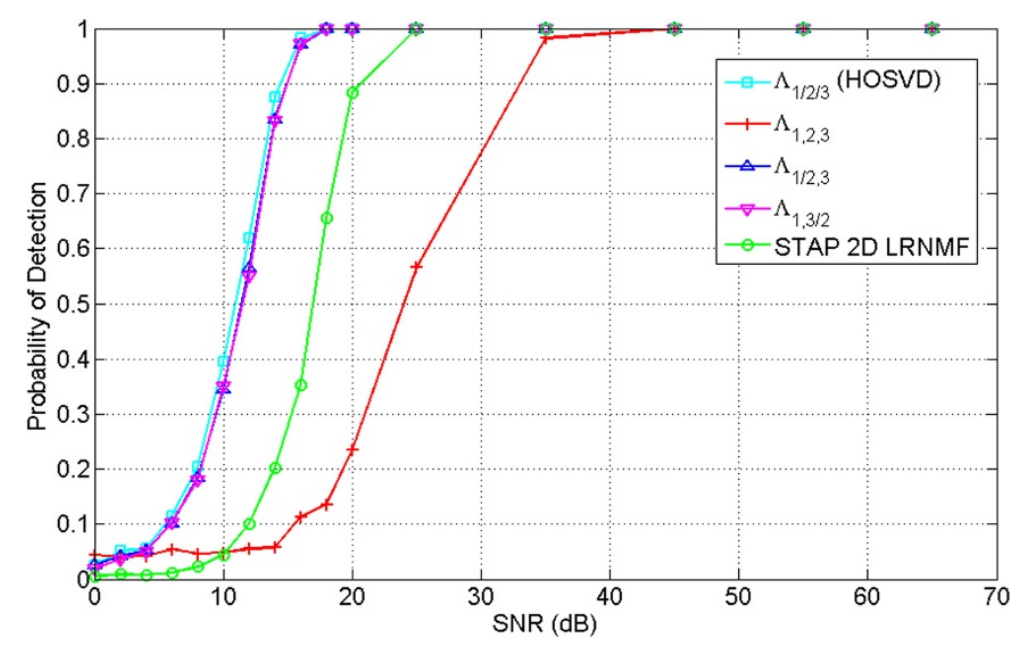

Figure 9 Pd versus SNR for $\boldsymbol{\rho}=\mathbf{1}, \boldsymbol{K}=\mathbf{3 0}, \mathrm{CNR}=\mathbf{4 0} \mathrm{dB}, \mathrm{Pfa}=\mathbf{1 0}^{\mathbf{- 2}}, \boldsymbol{N}_{\text {rea }}=\mathbf{1}, \mathbf{0 0 0}$. Target located at position $\left(\theta=0^{\circ}, v=10 \mathrm{~m} \mathrm{~s}^{-1}\right)$. 


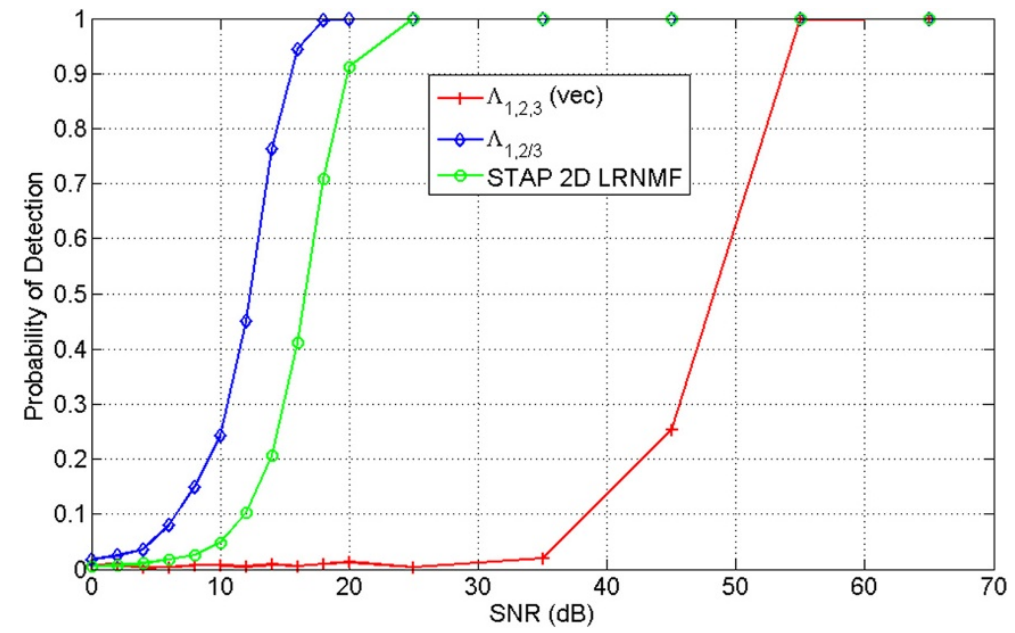

Figure 10 Pd versus SNR for $\rho=\mathbf{0 . 5}, K=\mathbf{3 0}, \mathrm{CNR}=\mathbf{4 0} \mathrm{dB}, \mathrm{Pfa}=10^{-\mathbf{2}}, \boldsymbol{N}_{\text {rea }}=\mathbf{1}, \mathbf{0 0 0}$. Target located at position $\left(\theta=0^{\circ}, \mathrm{V}=10 \mathrm{~m} \mathrm{~s}^{-1}\right)$.

the clutter is exploited by our filters, since $r_{3}$ is deficient. When the target is in the clutter ridge, the SINR loss is higher (especially for $\hat{\mathcal{W}}_{\operatorname{lr}(1 / 2 / 3)}, \hat{\mathcal{W}}_{\operatorname{lr}(1 / 2,3)}$, and $\left.\hat{\mathcal{W}}_{\operatorname{lr}(1,3 / 2)}\right)$ than the 2D LR STAP filter. By contrast, when $\rho=0.5$, the 2D LR STAP filter outperforms $\hat{\mathcal{W}}_{\operatorname{lr}(1,2 / 3)} \hat{\mathcal{W}}_{\operatorname{lr}(1,2,3)}$ (in the context of large $K$ ), since $r_{3}$ is full.

Results on Pfa and Pd The Pfa as a function of threshold is presented in Figures 7 and 8 . The probability of detection as a function of SNR is presented in Figures 9 and 10 for $K=30$. The thresholds are chosen in order to have a PfA of $10^{-2}$ according to Figures 7 and 8 . When $\rho=1, \hat{\Lambda}_{l r(1 / 2 / 3)}, \hat{\Lambda}_{l r(1 / 2,3)}$, and $\hat{\Lambda}_{l r(1,3 / 2)}$, which can only be obtained by AU-HOSVD, outperform $\hat{\Lambda}_{l r(1,2,3)}$ and the 2D STAP LRNMF. For instance, Pd is equal to $90 \%$ when the SNR is equal to $15 \mathrm{~dB}$ for $\hat{\Lambda}_{\operatorname{lr}(1 / 2 / 3)}, \hat{\Lambda}_{\operatorname{lr}(1 / 2,3)}$, and $\hat{\Lambda}_{l r(1,3 / 2)} ; 20 \mathrm{~dB}$ for the 2D STAP LRNMF; and $33 \mathrm{~dB}$ for $\hat{\Lambda}_{l r(1,2,3)}$. When $\rho=0.5, \hat{\Lambda}_{\operatorname{lr}(1,2 / 3)}$ outperforms $\hat{\Lambda}_{\operatorname{lr}(1,2,3)}$ and the 2D STAP LRNMF. For instance, Pd is equal to $90 \%$ when the SNR is equal to $16 \mathrm{~dB}$ for $\hat{\Lambda}_{l r(1,2 / 3)}, 20 \mathrm{~dB}$ for the 2D STAP LRNMF, and $54 \mathrm{~dB}$ for $\hat{\Lambda}_{l r(1,2,3) \text {. }}$

The results on Pd confirm the results on SINR loss concerning the most efficient partition for the two scenarios. In particular, it shows that the best results are provided by the filters and detectors which can only be obtained with the AU-HOSVD.

\section{Conclusion}

In this paper, we introduced a new multilinear decomposition: the AU-HOSVD. This new decomposition generalizes the HOSVD and highlights the correlated data in a multidimensional set. We showed that the properties of the AU-HOSVD are proven to be the same as those for HOSVD: the orthogonality and the LR decomposition. We have also derived LR filters and LR detectors based on
AU-HOSVD for multidimensional data containing one LR structure contribution. Finally, we applied our new LR filters and LR detectors to polarimetric space-time adaptive processing (STAP) where the dimension of the problem is three and the contribution of the background is correlated in time and space. Simulations based on signal-tointerference-plus-noise ratio (SINR) losses, probability of detection (Pd), and probability of false alarm (Pfa) showed the interest of our approach: LR filters and LR detectors which can be obtained only from AU-HOSVD outperformed the vectorial approach and those obtained from HOSVD in the general polarimetry physic model (where the channels $\mathrm{HH}$ and VV are not completely correlated). The main future work concerns the application of the LR filters and LR detectors developed from the AU-HOSVD for the general system of MIMO-STAP [23-26].

\section{Endnotes}

${ }^{a}$ This new decomposition has similarity with the block term decomposition introduced in [13,32] and [33], which proposes to unify HOSVD and CP.

${ }^{\mathrm{b}}$ Using this assumption, a low-rank vector STAP filter can be derived based on the projector onto the subspace orthogonal to the clutter (see $[10,11,34]$ for more details).

${ }^{\mathrm{c}}$ This definition of rank is directly extended from the definition of $p$-ranks.

\section{Appendices}

\section{Appendix 1}

\section{Proof of Proposition 4.1}

By analogy with the vector case [35], we derive the optimal filter $\mathcal{W}_{\text {opt }}$, which maximizes the output SINR $_{\text {out: }}$ :

$$
\operatorname{SINR}_{\text {out }}=\frac{\left|<\mathcal{W}_{\text {opt }}, \mathcal{S}>\right|^{2}}{\operatorname{vec}\left(\mathcal{W}_{\text {opt }}\right)^{H} \operatorname{SqMat}(\mathcal{R}) \operatorname{vec}\left(\mathcal{W}_{\text {opt }}\right)} .
$$


Then,

$$
\begin{aligned}
& \left|<\mathcal{W}_{\text {opt }}, \mathcal{S}>\right|^{2} \\
= & \left|\operatorname{vec}\left(\mathcal{W}_{\mathrm{opt}}\right)^{H} \operatorname{vec}(\mathcal{S})\right|^{2} \\
= & \left|\operatorname{vec}\left(\mathcal{W}_{\mathrm{opt}}\right)^{H} \operatorname{SqMat}(\mathcal{R})^{\frac{1}{2}} \operatorname{SqMat}(\mathcal{R})^{-\frac{1}{2}} \operatorname{vec}(\mathcal{S})\right|^{2} \\
= & \left|<\operatorname{SqMat}(\mathcal{R})^{\frac{1}{2}(H)} \operatorname{vec}\left(\mathcal{W}_{\mathrm{opt}}\right), \operatorname{SqMat}(\mathcal{R})^{-\frac{1}{2}} \operatorname{vec}(\mathcal{S})>\right|^{2}
\end{aligned}
$$

By Cauchy-Schwarz inequality, (47) is maximum when $\operatorname{SqMat}(\mathcal{R})^{\frac{1}{2}(H)} \operatorname{vec}\left(\mathcal{W}_{\text {opt }}\right)=\operatorname{SqMat}(\mathcal{R})^{-\frac{1}{2}} \operatorname{vec}(\mathcal{S})$ and $\operatorname{vec}\left(\mathcal{W}_{\text {opt }}\right)=\operatorname{SqMat}(\mathcal{R})^{-1} \operatorname{vec}(\mathcal{S})$. We replace $\mathcal{W}_{\text {opt }}$ in (46):

x'SINR

$$
\begin{aligned}
& =\frac{\left|\operatorname{vec}(\mathbf{S})^{H} \operatorname{SqMat}(\mathcal{R})^{-1} \operatorname{vec}(\mathbf{S})\right|^{2}}{\operatorname{vec}(\mathbf{S})^{H} \operatorname{SqMat}(\mathcal{R})^{-1} \operatorname{SqMat}(\mathcal{R}) \operatorname{SqMat}(\mathcal{R})^{-1} \operatorname{vec}(\mathbf{S})} \\
& =\left|\operatorname{vec}(\mathbf{S})^{H} \operatorname{SqMat}(\mathcal{R})^{-1} \operatorname{vec}(\mathbf{S})\right| \\
& =\operatorname{SINR}_{\max }
\end{aligned}
$$

\section{Appendix 2}

\section{Proof of Proposition 4.2}

We propose to derive the low-rank tensor filter as follows:

- First, the covariance tensor $\mathcal{R}$ is decomposed with the AU-HOSVD:

$$
\begin{array}{r}
\mathcal{R}=\mathcal{K}_{\mathbb{A}_{1} / \ldots / \mathbb{A}_{2 L}} \times \mathbb{A}_{1} \mathbf{U}^{\left(\mathbb{A}_{1}\right)} \ldots \times_{\mathbb{A}_{L}} \mathbf{U}^{\left(\mathbb{A}_{L}\right)} \\
\times_{\mathbb{A}_{L+1}} \mathbf{U}^{\left(\mathbb{A}_{1}\right) *} \ldots \times_{\mathbb{A}_{2 L}} \mathbf{U}^{\left(\mathbb{A}_{L}\right) *} .
\end{array}
$$

- $r_{\mathbb{A}_{1}}, \ldots, r_{\mathbb{A}_{L}}\left(r_{\mathbb{A}_{l}}=\operatorname{rank}\left([\mathcal{R}]_{\mathbb{A}_{l}}\right)\right)$ are estimated.

- Each $\mathbf{U}^{\left(\mathbb{A}_{l}\right)}$ is truncated to obtain

$$
\mathbf{U}_{0}^{\left(\mathbb{A}_{l}\right)}=\left[\mathbf{u}_{r_{\mathbb{A}_{l}}+1}^{\left(\mathbb{A}_{l}\right)}, \ldots, \mathbf{u}_{\mathbb{A}_{l}}^{\left(\mathbb{A}_{l}\right)}\right]
$$

- Proposition 3.2, with $\mathcal{H}=\boldsymbol{X}, \mathcal{H}_{c}=\mathcal{C}$ and $\mathcal{H}_{0}=\alpha \mathcal{S}+\mathcal{N}$ is applied in order to remove the LR contribution:

$$
\mathcal{X} \times_{\mathbb{A}_{1}} \mathbf{U}_{0}^{\left(\mathbb{A}_{1}\right)} \mathbf{U}_{0}^{\left(\mathbb{A}_{1}\right) H} \ldots \times_{\mathbb{A}_{L}} \mathbf{U}_{0}^{\left(\mathbb{A}_{L}\right)} \mathbf{U}_{0}^{\left(\mathbb{A}_{L}\right) H} \approx \alpha \mathcal{S}+\mathcal{N} .
$$

- The problem is then to filter $\mathcal{S}$ which is corrupted by a white noise $\mathcal{N}$. The filter given by (27) is applied with $\mathcal{R}=\mathfrak{J}_{I_{1} \ldots I_{p}}$ :

$y=\left|<\mathcal{S}, \boldsymbol{X} \times_{\mathbb{A}_{1}} \mathbf{U}_{0}^{\left(\mathbb{A}_{1}\right)} \mathbf{U}_{0}^{\left(\mathbb{A}_{1}\right) H} \ldots \times_{\mathbb{A}_{L}} \mathbf{U}_{0}^{\left(\mathbb{A}_{L}\right)} \mathbf{U}_{0}^{\left(\mathbb{A}_{L}\right) H}>\right|$.
- Finally, the output filter is rewritten:

$$
\begin{aligned}
& \mathcal{W}_{\operatorname{lr}\left(\mathbb{A}_{1}, \ldots, \mathbb{A}_{P}\right)}=\mathcal{S} \times \times_{\mathbb{A}_{1}} \mathbf{U}_{0}^{\left(\mathbb{A}_{1}\right)} \mathbf{U}_{0}^{\left(\mathbb{A}_{1}\right) H} \ldots \\
& \times_{\mathbb{A}_{L}} \mathbf{U}_{0}^{\left(\mathbb{A}_{L}\right)} \mathbf{U}_{0}^{\left(\mathbb{A}_{L}\right) H} \\
& y=\left|<\mathcal{W}_{\mathbb{A}_{1}, \ldots, \mathbb{A}_{P}}, \boldsymbol{X}>\right|
\end{aligned}
$$

\section{Appendix 3}

\section{Proof of Proposition 4.3}

To prove Proposition 4.3, let us recall the hypothesis test:

$$
\left\{\begin{array}{l}
H_{0}: \mathcal{X}=\mathcal{C}+\mathcal{N}, \boldsymbol{X}_{k}=\mathcal{C}_{k}+\mathcal{N}_{k}, k \in[1, K] \\
H_{1}: \mathcal{X}=\alpha \mathcal{S}+\mathcal{C}+\mathcal{N}, \boldsymbol{X}_{k}=\mathcal{C}_{k}+\mathcal{N}_{k}, k \in[1, K]
\end{array}\right.
$$

- Using Proposition 3.2, the data are preprocessed in order to remove the LR contribution. We denote $\mathbb{A}_{1} \ldots \mathbb{A}_{L}=\times_{\mathbb{A}_{1}} \mathbf{U}_{0}^{\left(\mathbb{A}_{1}\right)} \mathbf{U}_{0}^{\left(\mathbb{A}_{1}\right) H} \ldots \times_{\mathbb{A}_{L}} \mathbf{U}_{0}^{\left(\mathbb{A}_{L}\right)} \mathbf{U}_{0}^{\left(\mathbb{A}_{L}\right) H}$. The hypothesis test becomes

$$
\left\{\begin{aligned}
H_{0}: & x_{\mathbb{A}_{1} \ldots \mathbb{A}_{L}}=\mathcal{N}_{\mathbb{A}_{1} \ldots \mathbb{A}_{L}}, x_{k, \mathbb{A}_{1} \ldots \mathbb{A}_{L}}=\mathcal{N}_{k, \mathbb{A}_{1} \ldots \mathbb{A}_{L}} \\
H_{1}: & x_{\mathbb{A}_{1} \ldots \mathbb{A}_{L}}=\alpha \mathcal{S}_{\mathbb{A}_{1} \ldots \mathbb{A}_{L}}+\mathcal{N}_{\mathbb{A}_{1} \ldots \mathbb{A}_{L}}, \\
& x_{k, \mathbb{A}_{1} \ldots \mathbb{A}_{L}}=\mathcal{N}_{k, \mathbb{A}_{1} \ldots \mathbb{A}_{L}}
\end{aligned}\right.
$$

- Then, the operator vec is applied, which leads to

$$
\left\{\begin{aligned}
H_{0}: & \operatorname{vec}\left(\boldsymbol{X}_{\mathbb{A}_{1} \ldots \mathbb{A}_{L}}\right)=\operatorname{vec}\left(\mathcal{N}_{\mathbb{A}_{1} \ldots \mathbb{A}_{L}}\right) \\
& \operatorname{vec}\left(X_{k, \mathbb{A}_{1} \ldots \mathbb{A}_{L}}\right)=\operatorname{vec}\left(\mathcal{N}_{k, \mathbb{A}_{1} \ldots \mathbb{A}_{L}}\right) \\
H_{1}: & \operatorname{vec}\left(\boldsymbol{X}_{\mathbb{A}_{1} \ldots \mathbb{A}_{L}}\right)=\operatorname{vec}\left(\mathcal{S}_{\mathbb{A}_{1} \ldots \mathbb{A}_{L}}\right)+\operatorname{vec}\left(\mathcal{N}_{\mathbb{A}_{1} \ldots \mathbb{A}_{L}}\right) \\
& \operatorname{vec}\left(X_{k, \mathbb{A}_{1} \ldots \mathbb{A}_{L}}\right)=\operatorname{vec}\left(\mathcal{N}_{k, \mathbb{A}_{1} \ldots \mathbb{A}_{L}}\right)
\end{aligned}\right.
$$

where $\operatorname{vec}\left(\mathcal{N}_{\mathbb{A}_{1} \ldots \mathbb{A}_{L}}\right), \operatorname{vec}\left(\mathcal{N}_{k, \mathbb{A}_{1} \ldots \mathbb{A}_{L}}\right) \sim \mathcal{C N}\left(\mathbf{0}, \sigma^{2} \mathbf{I}_{I_{1} \ldots I_{p}}\right)$.

- The problem is then to detect a signal $\operatorname{vec}\left(\boldsymbol{S}_{\mathbb{A}_{1} \ldots \mathbb{A}_{L}}\right)$ corrupted by a white noise vec $\left(\mathcal{N}_{\mathbb{A}_{1} \ldots \mathbb{A}_{L}}\right)$. Since $\alpha$ and $\sigma$ are unknown, the adaptive normalized matched filter introduced in [30] can be applied:

$$
\begin{aligned}
& \Lambda_{\mathbb{A}_{1} \ldots \mathbb{A}_{L}} \\
& =\frac{\left|<\operatorname{vec}\left(\mathcal{S}_{\mathbb{A}_{1} \ldots \mathbb{A}_{L}}\right), \operatorname{vec}\left(\boldsymbol{X}_{\mathbb{A}_{1} \ldots \mathbb{A}_{L}}\right)>\right|^{2}}{<\operatorname{vec}\left(\boldsymbol{S}_{\mathbb{A}_{1} \ldots \mathbb{A}_{L}}\right), \operatorname{vec}\left(\boldsymbol{S}_{\mathbb{A}_{1} \ldots \mathbb{A}_{L}}\right)><\operatorname{vec}\left(\boldsymbol{X}_{\mathbb{A}_{1} \ldots \mathbb{A}_{L}}\right), \operatorname{vec}\left(\boldsymbol{X}_{\mathbb{A}_{1} \ldots \mathbb{A}_{L}}\right)>} .
\end{aligned}
$$

- Finally, the proposition is proven by applying the operator $\operatorname{vec}^{-1}$.

\section{Competing interests}

The authors declare that they have no competing interests.

\section{Author details}

${ }^{1}$ SATIE, ENS Cachan, 61, av President Wilson, Cachan 94230, France. ${ }^{2}$ SONDRA, Supelec, 3 rue Joliot-Curie, Gif-sur-Yvette 91192 , France. ${ }^{3}$ LISTIC - Polytech Annecy-Chambéry, 5, chemin de Bellevue, Annecy le Vieux 74944, France.

Received: 4 April 2014 Accepted: 14 July 2014

Published: 30 July 2014 


\section{References}

1. M Boizard, G Ginolhac, F Pascal, P Forster, A new tool for multidimensional low-rank STAP filter: cross HOSVDs, in Proceedings of EUSIPCO (Bucharest, Romania, September 2012)

2. T Kolda, B Bader, Tensor decompositions and applications. SIAM Rev. 51, 455-500 (2009)

3. LD Lathauwer, BD Moor, J Vandewalle, A multilinear singular value decomposition. SIAM J. Matrix Anal. Apl. 24(4), 1253-1278 (2000)

4. RA Harshman, Foundation of the PARAFAC procedure: model and conditions for an explanatory multi-mode factor analysis. UCLA Working Pap. Phon. 16, 1-84 (1970)

5. ND Sidiropoulos, R Bro, GB Giannakis, Parallel factor analysis in sensor array processing. IEEE Trans. Proc. Sig. Proc. 48(8), 2377-2388 (2000)

6. ND Sidiropoulos, R Bro, GB Giannakis, Blind PARAFAC receivers for DS-CDMA systems. IEEE Trans. Proc. Sig. Proc. 48(3), 810-823 (2000)

7. ALF de Almeida, G Favier, JCM Mota, Constrained Tucker-3 model for blind beamforming. Elsevier Signal Process. 89, 1240-1244 (2009)

8. G Favier, MN da Costa, ALF de Almeida, JMT Romano, Tensor space time (TST) coding for MIMO wireless communication systems. Elsevier Signal Process. 92, 1079-1092 (2012)

9. LR Tucker, Some mathematical notes on three-mode factor analysis. Psychometrika. 31, 279-311 (1966)

10. I Kirsteins, D Tufts, Adaptive detection using a low rank approximation to a data matrix. IEEE Trans. Aero. Elec. Syst. 30, 55-67 (1994)

11. A Haimovich, Asymptotic distribution of the conditional signal-to-noise ratio in an eigenanalysis-based adaptive array. IEEE Trans. Aero. Elec. Syst. 33, 988-997 (1997)

12. P Comon, Tensors : a brief introduction. IEEE Signal Process. Mag. 31(3), 44-53 (2014)

13. LD Lathauwer, Decompositions of a higher-order tensor in block termspart I: lemmas for partitioned matrices. SIAM J. Matrix Anal. Apl. 30(3), 1022-1032 (2008)

14. D Muti, S Bourennane, Multidimensional filtering based on a tensor approach. Elsevier Signal Process. 85, 2338-2353 (2005)

15. NL Bihan, G Ginolhac, Three-mode data set analysis using higher order subspace method: application to sonar and seismo-acoustic signal processing. Elsevier Signal Process. 84(5), 919-942 (2004)

16. M Haardt, F Roemer, GD Galdo, Higher-order SVD-based subspace estimation to improve the parameter estimation accuracy in multidimensionnal harmonic retrieval problems. IEEE Trans. Proc. Sig. Proc. 56(7), 3198-3213 (2008)

17. LD Lathauwer, BD Moor, J Vandewalle, Independent component analysis and (simultaneous) third-order tensor diagonalization. IEEE Trans. Sig. Proc. 49, 2262-2271 (2001)

18. MAO Vasilescu, D Terzopoulos, Multilinear subspace analysis of image ensembles, in Proc. of the IEEE Conf. on Computer Vision and Pattern Recognition, (June 2003)

19. J Salmi, A Richter, V Koivunen, Sequential unfolding SVD for tensors with applications in array signal processing. IEEE Trans. Sig. Proc. 57(12), 4719-4733 (2009)

20. J Ward, Space-time adaptive processing for airborne radar. Technical report. Lincoln Lab., MIT, Lexington, Mass., USA (December 1994)

21. LE Brennan, FM Staudaher, Subclutter visibility demonstration. Technical report. RL-TR-92-21, Adaptive Sensors Incorporated (March 1992)

22. G Showman, W Melvin, M Belenkii, Performance evaluation of two polarimetric STAP architectures, in Proc. of the IEEE Int. Radar Conf, (May 2003), p. 59-65

23. VF Mecca, D Ramakrishnan, JL Krolik, MIMO radar space-time adaptive processing for multipath clutter mitigation, in Fourth IEEE Workshop On Sensor Array and Multichannel Processing, 2006, (July 2006), p. 249-253

24. CY Chen, PP Vaidyanathan, A subspace method for MIMO radar space-time adaptive processing, in IEEE International Conference on ICASSP 2007 (Honolulu, Hawaii, USA, 2007), p. 925-928

25. CY Chen, PP Vaidyanathani, MIMO radar space-time adaptive processing using prolate spheroidal wave functions. IEEE Trans. Sig. Proc. 56(2), 623-635 (2008)

26. R Fa, RC de Lamare, P Clarke, Reduced-rank STAP for MIMO radar based on joint iterative optimization of knowledge-aided adaptive filters, in 2009 Conference Record of the Forty-Third Asilomar Conference On Signals, Systems and Computers, pp. 496-500
27. LD Lathauwer, BD Moor, JVandewalle, On the best rank-1 and rank- $\left(r_{1}, r_{2}, \ldots, r_{n}\right)$ approximation and applications of higher-order tensors. SIAM J. Matrix Anal. Apl. 21(4), 1324-1342 (2000)

28. S Miron, NL Bihan, J Mars, Vector-sensor MUSIC for polarized seismic sources localization. EURASIP J. Adv. Signal Process. 2005, 74-84 (2005)

29. IS Reed, JD Mallett, LE Brennan, Rapid convergence rate in adaptive arrays. IEEE Trans. Aero. Elec. Syst. AES-10(6), 853-863 (1974)

30. LL Scharf, B Friedlander, Matched subspace detector. IEEE Trans. Sig. Proc. 42(8), 2146-2157 (1994)

31. M Rangaswamy, FC Lin, KR Gerlach, Robust adaptive signal processing methods for heterogeneous radar clutter scenarios. Elsevier Signal Process. 84, 2004

32. LD Lathauwer, Decompositions of a higher-order tensor in block termspart II definitions and uniqueness. SIAM J. Matrix Anal. Apl. 30(3), 1033-1066 (2008)

33. LD Lathauwer, D Nion, Decompositions of a higher-order tensor in block terms - part III: alternating least squares algorithms. SIAM J. Matrix Anal. Apl. 30(3), 1067-1083 (2008)

34. G Ginolhac, P Forster, F Pascal, JP Ovarlez, Performance of two low-rank STAP filters in a heterogeneous noise. IEEE Trans. Signal Process. 61, 57-61 (2013)

35. LE Brennan, LS Reed, Theory of adaptive radar. IEEE Trans. Aero. Elec. Syst. 9(2), 237-252 (1973)

doi:10.1186/1687-6180-2014-119

Cite this article as: Boizard et al:: Low-rank filter and detector for multidimensional data based on an alternative unfolding HOSVD: application to polarimetric STAP. EURASIP Journal on Advances in Signal Processing 2014 2014:119.

\section{Submit your manuscript to a SpringerOpen ${ }^{\mathcal{O}}$ journal and benefit from:}

- Convenient online submission

- Rigorous peer review

- Immediate publication on acceptance

- Open access: articles freely available online

- High visibility within the field

- Retaining the copyright to your article

Submit your next manuscript at $\gg$ springeropen.com 